\title{
The Visuotopic Component of the Multisensory Map in the Deep Laminae of the Cat Superior Colliculus
}

\author{
M. Alex Meredith ${ }^{1}$ and Barry E. Stein ${ }^{2}$ \\ Departments of 'Anatomy and 'Physiology, Medical College of Virginia, Virginia Commonwealth University, Richmond, \\ Virginia 23298
}

\begin{abstract}
A well-defined map of visual space is located in the deep laminae of the cat superior colliculus. The horizontal meridian is oriented rostral-caudal, while the vertical meridian is oriented perpendicular to it in the rostral third of the structure. This map represents the entire contralateral visual field and extends approximately $40^{\circ}$ into ipsilateral visual space. Although the deep-laminae visuotopy is similar to that found in the superficial laminae of the same structure, the topographic register among these maps is most secure rostrally but becomes increasingly poorer at more caudal and lateral locations. The combination of 2 features distinguish the deeplayer visual representation from that found in the superficial laminae and in geniculocortical systems: (1) the constituent visual receptive fields are very large (mean diameter, $66.9^{\circ}$ ), and (2) the majority $(>70 \%)$ of the neurons composing it receive nonvisual inputs. Because the visual receptive fields of visual-multisensory neurons are significantly larger than those of neighboring neurons that respond only to visual stimuli, far more visual-multisensory neurons are activated by any given visual stimulus. These data, when coupled with those from previous studies, suggest that, from a functional perspective, deep-laminae visual neurons form one component of an integrated multisensory map, and that their topographic organization is essential for the normal dynamics of multisensory integration.
\end{abstract}

A feature common to the nervous systems of higher organisms is the presence of organized, maplike representations of sensory space or receptor epithelia. At each level of the neuraxis, the different sensory modalities occupy spatially distinct territories that are defined both functionally and cytoarchitectonically. In the cortex, and in some areas of thalamus, the domains of the various primary sensory representations are further separated from one another by intervening "secondary" maps (and in the cortex by "association" areas). These maps are distinguished from their adjoining "primary" counterparts by mirror-image reversals in the progression of their receptive fields and, usually, by their specialization for different submodality features, as well.

A notable exception to this plan is found in the deeper layers (ventral to the stratum opticum) of the superior colliculus. Here, neurons responsive to visual, auditory, and somatosensory stim-

\footnotetext{
Received July 31, 1989; revised July 20, 1990; accepted July 27, 1990.

The authors wish to thank J. Nelson and N. London for their technical assistance. This work was supported by NIH Grant NS 22543.

Correspondence should be addressed to $M$. Alex Meredith, Department of Anatomy, Medical College of Virginia, Virginia Commonwealth University, Box 709 MCV Station, Richmond, VA 23298-0709.

Copyright (C) 1990 Society for Neuroscience $0270-6474 / 90 / 113727-16 \$ 03.00 / 0$
}

uli, as well as to their various combinations, are intermixed, and no uninterrupted sheet of tissue is devoted to the representation of any single sensory modality. The significance of this "deviation" from the common scheme is believed to reflect the involvement of this structure in attentive and orientation responses to stimuli from any and all of these sensory modalities (Sprague and Meikle, 1965; Sprague, 1966). Overt responses, expressed by the orientation of a number of sensory organs (eyes, pinnae, head), are initiated via the extensive deep-laminae efferent system that projects to premotor areas of the brain stem and spinal cord controlling the position of these organs (for a review, see Huerta and Harting, 1984). Therefore, it has been suggested that the convergence and intermingling of sensory inputs represents an efficient means for the different sensory systems to gain access to a common motor pathway (e.g., Stein et al., 1976; Jay and Sparks, 1987). Even though the various multisensory and unimodal neurons do not reveal any obvious pattern of arrangement within the deep laminae, their sensory inputs show a high degree of topographic alignment in a variety of species (Gordon, 1973; Drager and Hubel, 1975; Stein et al., 1976; Tiao and Blakemore, 1976; Chalupa and Rhoades, 1977; Finlay et al., 1978; Stein and Dixon, 1978; Graham et al., 1981; Knudsen, 1982; King and Palmer, 1983, 1985; Middlebrooks and Knudsen, 1984; Meredith and Stein, 1986a). This alignment is not as surprising as it might seem: deep-laminae neurons are involved in initiating movements toward the specific spatial location from which stimuli originate, and, to avoid the initiation of competing or conflicting movements, their inputs should arrive from neural areas representing topographically related portions of sensory space.

That organized maps of visual and auditory space and the body surface exist in topographic register within the superior colliculus is well established. However, the known visuotopy is based primarily on information from the superficial laminae, where relatively small receptive fields (McIlwain and Buser, 1968; Feldon et al., 1970; Berman and Cynader, 1975; McIlwain, 1975), constructed by the convergence of inputs from the retina (Graybiel, 1975; Harting and Guillery, 1976) and primary visual cortex (Wickelgren and Sterling, 1969; Mcllwain and Fields, 1971; Rosenquist and Palmer, 1971; Stein and Arigbede, 1972; Berman and Cynader, 1975; Mize and Murphy, 1976; Ogasawara et al., 1984), compose the map (Apter, 1945; Feldon et al., 1970). Although it has been assumed that this superficial visual map bears some functional relationship to the nonvisual deep-laminae sensory representations, there is little data to support this assumption. Some superficial-deep connections have been reported on several species (Behan and Appell, 1987; Mooney et al., 1988a,b; Moschovakis et al., 1988; 
Rhoades et al., 1989), but there are also convincing data that superficial and deep divisions of the superior colliculus are functionally independent (Casagrande et al., 1972; Ogasawara et al., 1984). Furthermore, the comparatively refined elements of the superficial-laminae visuotopy (Feldon et al., 1970) that are dependent, in part, on primary corticotectal input are in stark contrast to the nature of the deep-layer representations of the nonvisual modalities, which are constituted by neurons with large receptive fields, coarse topographic relationships (Gordon, 1973; Stein et al., 1976; King and Palmer, 1983, 1985; Middlebrooks and Knudsen, 1984; Meredith and Stein, 1986a,b) and inputs from "extraprimary" pathways (Stein et al., 1983; Clemo and Stein, 1984, 1986; Segal and Beckstead, 1984; Meredith and Clemo, 1989).

Many studies have reported deep-laminae visually responsive neurons and have mapped their receptive fields (e.g., Stein and Arigbede, 1972; Gordon, 1973; Middlebrooks and Knudsen, 1984; Meredith and Stein, 1986a,b). While these receptive fields appear to be visuotopically organized, published information regarding deep-layer visual receptive fields is insufficient either to construct a map there or to assess its overall alignment with the other sensory representations in the superior colliculus. Therefore, the present series of experiments was initiated to explore the visuotopic organization within of these deep-laminae visual receptive fields, to compare these results to the visuotopy found in the superficial laminae, and to determine the incidence of nonvisual convergence on deep-laminae visual neurons.

An abstract describing some of these data was published previously (Meredith and Stein, 1988).

\section{Materials and Methods}

Data were obtained using standard extracellular recording techniques and have been described in detail in a previous report (Meredith and Stein, 1986b). Of significant concern in conducting the present experiments was ensuring that the procedures used preserved the integrity of the cortical regions controlling deep-layer visual activity, avoiding the suppressive effects of certain anesthetics on deep-laminae visual activity (Gordon, 1973), and stabilizing the alignment of visual and nonvisual axes. Furthermore, all procedures were performed in compliance with the Guide for the Care and Use of Laboratory Animals (NIH Publication 86-23) at Virginia Commonwealth University, which is accredited by the American Association for Accreditation of Laboratory Animal Care (AAALAC).

Surgial preparation. Approximately 1 week prior to the first recording session, each cat $(n=21)$ was anesthetized with sodium pentobarbital $(40 \mathrm{mg} / \mathrm{kg}$, with supplements of $5-10 \mathrm{mg} / \mathrm{kg}$, i.v.) and placed in a stereotaxic head-holder. Aseptic conditions were maintained while a $2-\mathrm{cm}$ craniotomy was made to expose the cortex dorsal to the superior colliculus, and a hollow cylinder/head-holding device (McHaffie and Stein, 1983) was implanted over this opening.

Despite the absence of wounds or pressure points, all recording experiments were conducted with anesthetized preparations. They were initiated with an anesthetic dose of ketamine hydrochloride $(30 \mathrm{mg} / \mathrm{kg}$, i.m.). The animal was intubated through the mouth, paralyzed (pancuronium bromide, $10 \mathrm{mg} / \mathrm{kg}$, i.v.; subsequent doses, $0.6-0.8 \mathrm{mg} / \mathrm{kg}$, i.v.) and ventilated with $25 \% \mathrm{O}_{2}$ and $75 \% \mathrm{~N}_{2} \mathrm{O}$. Subsequent doses of anesthetic (15-10 mg/kg/hr, i.v.), sufficient to maintain anesthesia in nonparalyzed preparations, were delivered routinely. Furthermore, this regimen was checked periodically during each experiment by permitting the animal to recover from paralysis. Expiratory $\mathrm{CO}_{2}$ was kept between 3.7 and $4.5 \%$, and body temperature was maintained at $37-38^{\circ} \mathrm{C}$ with a heating pad. The pupils were dilated with $1 \%$ atropine sulfate, and the positions of the optic disks were projected onto a $91-\mathrm{cm}$-diameter translucent hemisphere scribed in polar coordinates. The refractive error of the contralateral eye was corrected with a contact lens, and the ipsilateral eye was occluded.

The recording well was fit with a calibrated $x-y$ slide for the positioning of each electrode penetration, and the activity of single neurons was recorded with glass-insulated tungsten electrodes (impedance, $>1 \mathrm{M} \Omega$ at $1 \mathrm{kHz}, 12-20-\mu \mathrm{m}$ tip exposure). The depth of each neuron studied was noted, and successful recording penetrations were marked with electrolytic lesions $(12 \mathrm{~mA}, 15 \mathrm{sec})$ so that the positions of recorded neurons could be located histologically.

At the end of an experiment, paralytics, and then anesthetics, were discontinued. Each animal was then weaned from the ventilator and returned to its home cage after regaining respiratory and locomotive function. At the conclusion of a series of recording sessions (usually no greater than 4), each animal was killed with an overdose of barbiturate and perfused with physiological saline and $10 \%$ formalin. The midbrain was blocked and was processed using routine histological procedures (50- $\mu \mathrm{m}$ frozen sections, cresyl violet staining). Neuronal locations were determined to the nearest percent of the anterior--posterior (AP) and mediolateral (ML) extent of the superior colliculus (e.g., $45 \%$ from caudal end, $67 \%$ from midline) and transferred to a standardized representation of a horizontal section through the stratum griseum intermediale, as determined by acetylcholinesterase staining (Dunning et al., 1987).

Evaluation of sensory convergence and receptive-field mapping. Visually responsive neurons were sought using moving slits or spots of light projected directly onto the translucent hemisphere. Once a visually activated neuron was identified, its responsiveness to inputs from other modalities was tested using a variety of auditory (hisses, claps, whistles) and somatosensory (taps, brushes across the skin or hair with a camelhair brush, airpuffs) stimuli. If a neuron was unresponsive to these stimuli presented alone, the possibility of subthreshold inputs from these modalities was tested by presenting electronically generated visual-auditory and visual-somatosensory combinations at various temporal intervals (see Meredith et al., 1987). Visual neurons that were either excited or inhibited by stimuli from other modalities were classified as "visual-multisensory," while those neurons unaffected by other stimulus modalities were considered "visual-unimodal."

Visual receptive-field borders were mapped by moving a spot of light ( $1-6^{\circ}$ in diameter, generated by a Keeler Pantoscope) from the periphery inward from all directions until an enclosed responsive area was delimited directly on the translucent hemisphere. These receptive fields were later transferred from the hemisphere to a graphic representation of the visual field (for storage and analysis) that was marked in the same, polar coordinate system. Next, a series of brief qualitative tests were conducted to determine the general visual characteristics of the neuron (e.g., preferences for flashed or moving stimuli, selectivity for velocity, direction of movement, stimulus size, the presence of inhibitory surrounds, etc.).

Data analysis. For each neuron, the receptive-field diameter was determined by averaging the long and short diameters of the receptive field as measured on the hemisphere. To evaluate the mapping of points of elevation and azimuth within the visual field onto the deep laminae (below the stratum opticum), each graphically recorded visual receptive field was transformed from polar coordinates (as shown in Fig. 1A) to double-pole coordinates of constant elevation and azimuth (as depicted in Fig. $1 B$ ). The deep-layer visuotopic map was constructed by plotting first the anatomical location (e.g., AP and ML position) of each recording penetration on a representative horizontal section through the stratum griseum intermediale. Next, each recording locus was categorized according to the proportion $(0 \%,>0 \%$ but $<75 \%, \geq 75 \%)$ of receptive fields per penetration that included a specific, predetermined point picked from the double-pole representation of visual space. These selected points were from $-90^{\circ}$ to $+60^{\circ}$ elevation at intervals $90,60,30,0$, and $-10^{\circ}$ azimuth. Finally, penetrations in which similar proportions (e.g., $>75 \%$ ) of receptive fields per penetration encompassed a common point were grouped to determine the focus ( $>75 \%$ was defined as the "best" point image) and extent ( $>0 \%$ was defined as the point image; see Mcllwain, 1975, 1976) of the representation of different points in the visual field. The area of each point image and best point image was measured on the standardized horizontal section of the superior colliculus using a Zeiss MOP-3 digital planimeter.

To quantify and analyze statistically the relationship between the location of visual receptive fields and the anatomical position of their cell bodies, as well as to examine the relationship between the superficial and deep visuotopies, it was necessary to transform the visual field from polar coordinates (Fig. $1 C$ ) to a linear, Cartesian system of coordinates. This process would be similar to plotting the circular polar-coordinate representation of the visual field on a sheet of latex, then stretching the sheet (at the $45^{\circ}, 135^{\circ}, 225^{\circ}$, and $315^{\circ}$ radians) so that the circle became a square. In this manner, lines intersecting the horizontal axis become 
A.
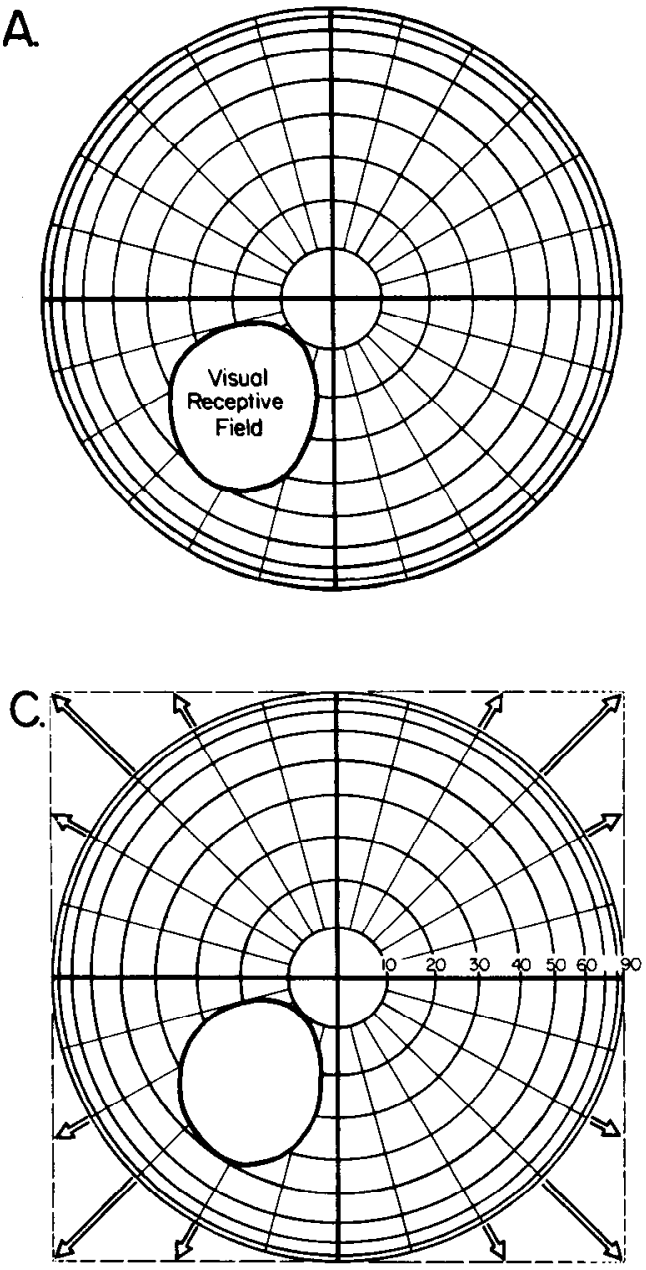

B.
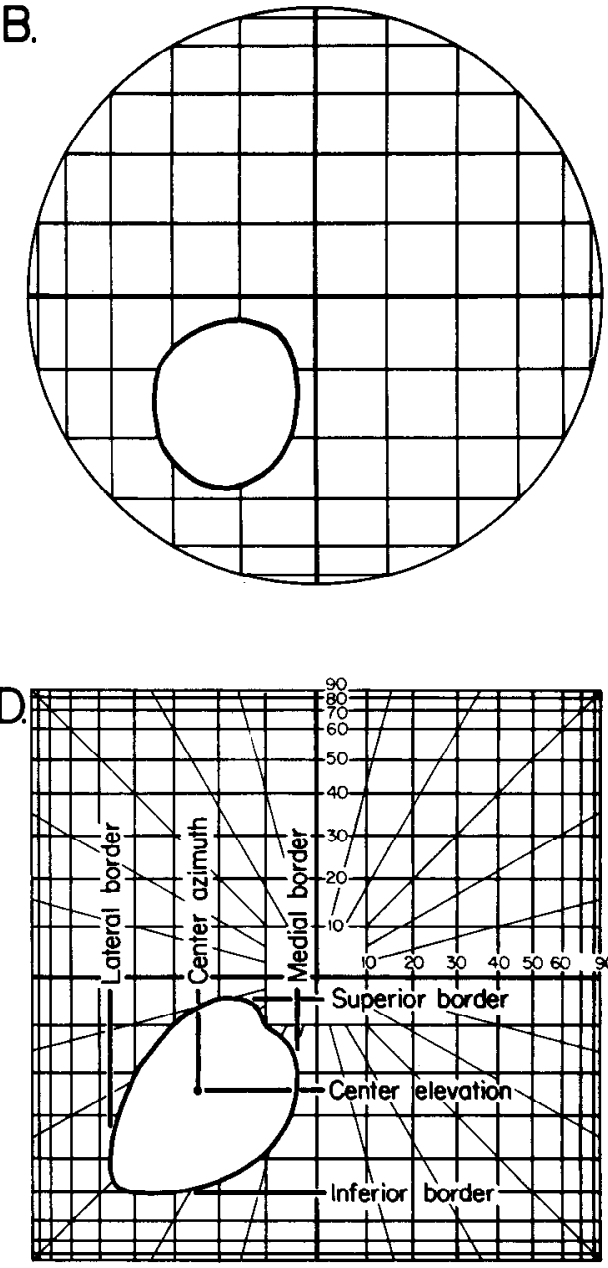

Figure 1. Coordinate systems used to record and measure receptive fields within visual field. $A$, Visual space and visual receptive fields were graphically recorded using a polar coordinate system. $B$, Qualitative assessment of a deep-laminae visuotopy was accomplished by transforming the visual field and accompanying visual receptive fields onto a double-pole coordinate system of constant elevation and azimuth. $C$ and $D$, Quantitative evaluation of the relationship of receptive field to neuronal location, as well as comparison of superficial-deep visuotopies, required transformation of polar $(C)$ to Cartesian coordinates $(D)$, where increasing eccentricity from the visual axis yielded independent and monotonically increasing values for $x$ (azimuth) and $y$ (elevation). The receptive-field measures that were examined (lateral, medial, superior and inferior borders, center elevation, and center azimuth) are denoted. See Materials and Methods, Data Analysis, for further details. perpendicular to it and represent lines of constant azimuth that increase monotonically in value as they progress temporally, as shown in Figure $1 D$. Similarly, lines orthogonal to the vertical axis represent lines of constant elevation that also increase monotonically in value as they progress along the vertical axis (see Fig. $1 D$ ). Receptive fields were graphically transferred from polar to Cartesian coordinates by imposing the radians from the polar coordinate system onto the $x-y$ system. Thus, a point in polar coordinates such as $30^{\circ}$ excursion along the $15^{\circ}$ radian could be transferred to the $x-y$ system at the intersection of the $15^{\circ}$ radian with $30^{\circ}$ line of elevation, and so on. This transformation of the visual field provided an advantage over other coordinate systems, because changes in elevation or azimuth were independent of one another. Furthermore, increases in the value of either of these measures were monotonic at the periphery as well as at the center of the field, a factor critical to the quantitative examination of the deep-layer visual representation. Quantification consisted of, first, determining a series of receptive-field measures that were indicators of a given receptive field's location in visual space, including its elevation, azimuth, and center. The elevation (in degrees, see Fig. $1 D$ ) of the receptive field was determined by projecting its superiormost and inferiormost border across the vertical meridian. Similarly, the most medial and lateral extent of the receptive field was extended across the horizontal meridian to measure its azimuth. Receptive-field centers were calculated as $1 / 2$ the distance between superior-inferior (central elevation) and medial-lateral (central azimuth) borders. These values were then matched with the anatomical location (AP, ML, depth, lamina) and modality (visualunimodal, visual-auditory, visual-somatosensory, or visual-auditorysomatosensory) of the neuron from which the receptive field was recorded. The data were pooled into a data file for regression analysis, $t$ tests, and analysis of covariance (Morrison, 1976).

\section{Results}

The primary aim of these experiments was to evaluate the deeplaminae visual topography of the cat superior colliculus. Nevertheless, each neuron also was carefully evaluated to determine the sensory modality(ies) to which it responded (i.e., visual, auditory, somatosensory, multisensory) and the lamina in which it was located. This allowed a comparison to be made of the sizes, topographies, and distributions of the receptive fields of

Table 1. Variation in visual receptive fields

\begin{tabular}{lcc} 
& $\begin{array}{c}\text { Mean diameter } \\
\pm \text { SD (degrees) }\end{array}$ & $\begin{array}{l}\text { Range } \\
\text { (degrees) }\end{array}$ \\
\hline Superticial (147) & $16.4 \pm 12.1$ & $2-81$ \\
Deep & & \\
$\quad$ All (254) & $66.9 \pm 27.3$ & $13.5-150.5$ \\
$\quad$ Unimodal (72) & $48.5 \pm 26.7$ & $13.5-150.5$ \\
$\quad$ Multimodal (182) & $74.1 \pm 23.9$ & $21-147.5$ \\
$\quad$ Visual-auditory (100) & $72.3 \pm 21.8$ & $31-141.5$ \\
$\quad$ Visual-somatosensory (51) & $70.2 \pm 25.6$ & $21-128$
\end{tabular}

Visual-auditory-somatosensory (31)

$86.5 \pm 25.6 \quad 38.5-147.5$

Values in parentheses represent number of neurons per category. 


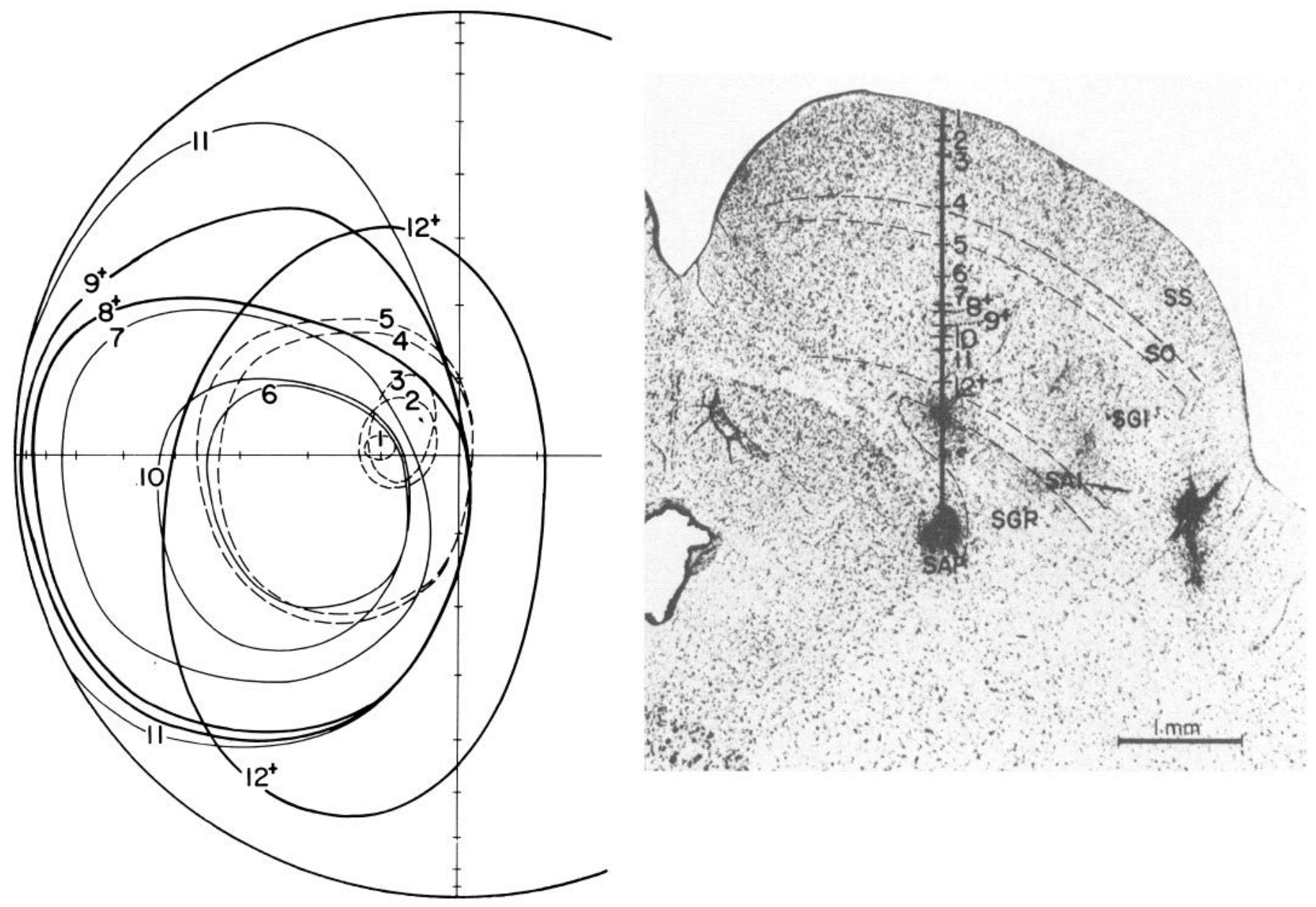

Figure 2. Visual receptive fields (left) mapped in single electrode penetration through superior colliculus (right). Dashed lines indicate visual receptive fields of superficial-layer neurons, solid lines represent those for deep-layer neurons, and pluses indicate deep-layer visual neurons that also receive nonvisual inputs. Receptive fields are numbered to correspond to neuronal positions along the electrode track. $S S$, strata superficiale (includes stratum zonale and stratum griseum superficiale); $S O$, stratum opticum; $S G I$, stratum griseum intermediale; $S A I$, stratum album intermediale; $S G P$, stratum griseum profundum; $S A P$, stratum album profundum.

a variety of visually responsive neurons. The visual receptive fields of 401 neurons were mapped in 21 cats $(64$ electrode penetrations through superficial and deep layers). As reported previously, superficial-laminae neurons $(n=147)$ were exclusively visual (Stein et al., 1976; Meredith and Stein, 1986b) and were organized into a detailed visuotopy (Feldon et al., 1970). No attempts were made to add further detail to the existing maps of these uppermost laminae; the map presented here was constructed from the receptive fields of unimodal and multisensory neurons $(n=254)$ that were located ventral to the stratum opticum.

\section{Receptive-field variation along an electrode track}

Characteristic examples of visual receptive fields that were encountered at different depths along a single electrode penetration are illustrated in Figure 2. As in most electrode penetrations, the visual receptive fields exhibited considerable variation in size, but those of deep-laminae neurons were consistently larger than those of the superficial-laminae neurons (see Table 1). Yet, regardless of size, receptive fields in the same electrode penetration tended to overlap one another, thereby exhibiting a coarse, laminar-independent visuotopic "registry."

During a typical electrode penetration, 2 response features appeared to change abruptly at the superficial-deep-laminae border: individual receptive fields increased dramatically in size, and there was now a high probability $(>70 \%)$ that a visually responsive neuron would also respond to nonvisual stimuli. The increment in size was so large that regardless of the neuron type (i.e., visual, visual-auditory, visual-somatosensory, trimodal), the diameters of the deep-layer visual receptive fields averaged a 4-fold increase over their superficial-laminae counterparts (see Table 1). Comparisons of pairs $(n=17)$ of subjacent neurons separated by the lower border of the stratum opticum (but within $500 \mu \mathrm{m}$ of one another) showed a large (207\%), statistically significant (paired $t$ test, $p<0.001$ ) increase in receptive-field diameter $\left(22.6 \pm 17.9^{\circ}\right.$ for stratum opticum neurons vs. 46.8 $\pm 24.7^{\circ}$ for upper stratum griseum intermediate neurons).

Despite the changes in receptive-field size and sensory convergence that occurred at the superficial-deep-laminae border, neither of these properties showed any consistent (e.g., monotonic) relationship with depth within the deep laminae. Unimodal and multisensory neurons appeared randomly intermixed throughout the deep laminae, and receptive-field size showed only a weak correlation with depth (linear regression, $\mathrm{r}$ $=-0.255$; see Fig. $3 A$ ). Instead, several features appeared to be covarying: (1) visually responsive neurons became progres- 
A.

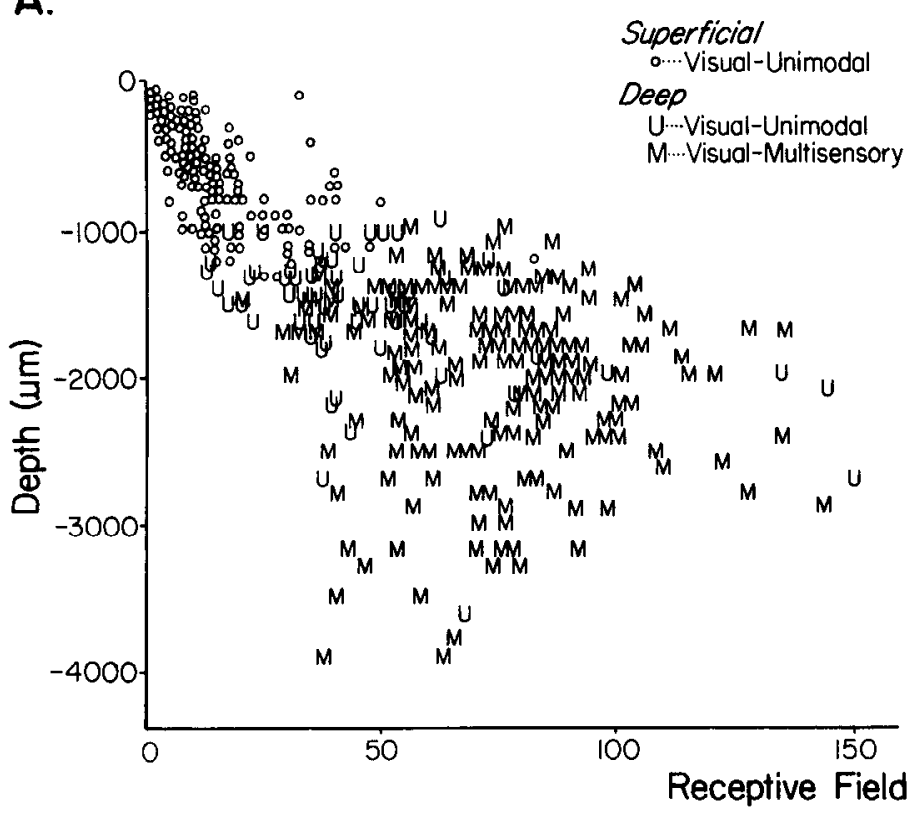

B.

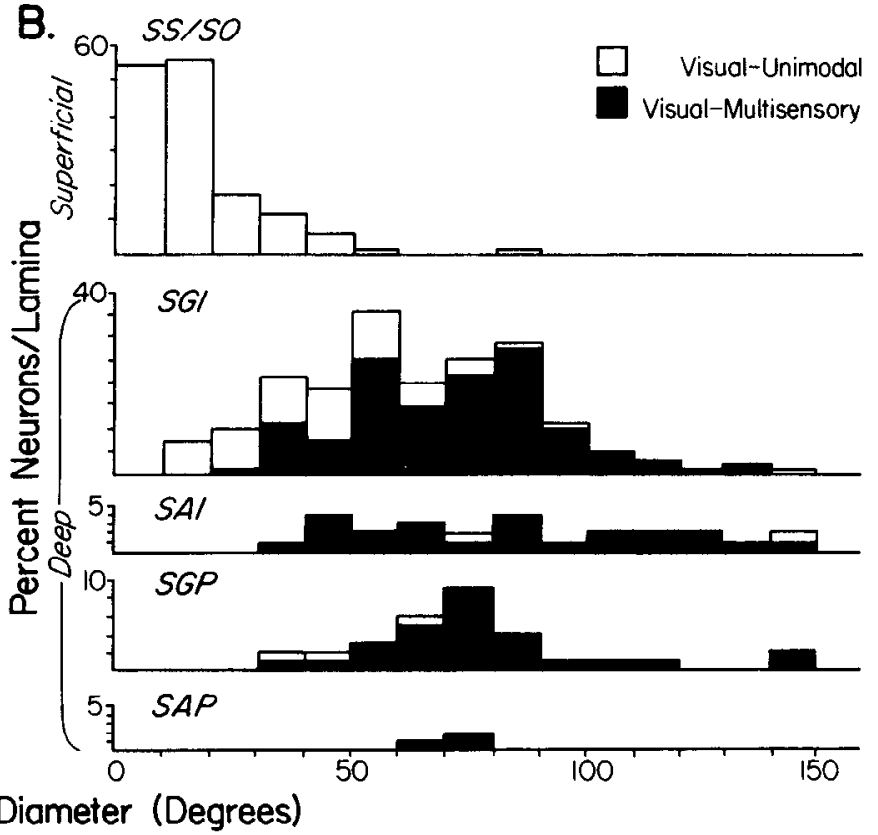

Figure 3. Relationship of receptive-field size and neuronal depth within superior colliculus. $A$, Receptive fields increased in size from superficial to deep laminae and also increased within the deep laminae as a function of the incidence of nonvisual inputs. However, there was little correlation $(r=0.255)$ among receptive-field diameter and neuronal depth within the deep laminae. $B$, The distribution of receptive-field diameters within each of the superior colliculus laminae and the proportions and sizes of visual-unimodal versus visual-multisensory neurons are shown by the histograms. For abbreviations, see Figure 2.

sively less frequent below the stratum griseum intermediale, so that the sample decreased with depth; (2) regardless of depth, peripheral receptive fields tended to be larger than central ones (analysis of covariance, $p<0.001$ ); and (3) multisensory neurons had significantly ( $t$ test; $p<0.001)$ larger receptive fields than did unimodal neurons. Because fewer than $14 \%$ of the deeplayer visual neurons were found in or below the stratum griseum profundum, most of the data were derived from intermediatelaminae neurons, and these data are summarized in Figure 3.

\section{Deep-layer visuolopy: receptive-field considerations}

From qualitative observations, it was obvious that deep-layer neurons rostral in the superior colliculus generally had their receptive fields near the projection of the area centralis, while caudal neurons had their receptive fields in inferior-temporal visual space. Similarly, medial neurons had superior receptive fields, and lateral neurons had inferior receptive fields (Fig. 4). In order to quantify the relationship between neuron location and receptive-field position, the center and various borders (superior, inferior, medial, and lateral) of each receptive field were plotted against the neuron's histologically determined location (Fig. 5). Each of the receptive-field measures (center or a particular border) used in this analysis proved to be effective and

Figure 4. Receptive fields mapped during 7 different electrode penetrations within same superior colliculus showed general and predictable shift in relation to recording site. The position of each electrode penetration is indicated on the dorsal view of the superior colliculus (center), and the corresponding receptive fields are indicated within the depictions of the visual field. Rostral electrode penetrations yielded superficial unimodal (solid black), deep unimodal (light line), and deep multisen-

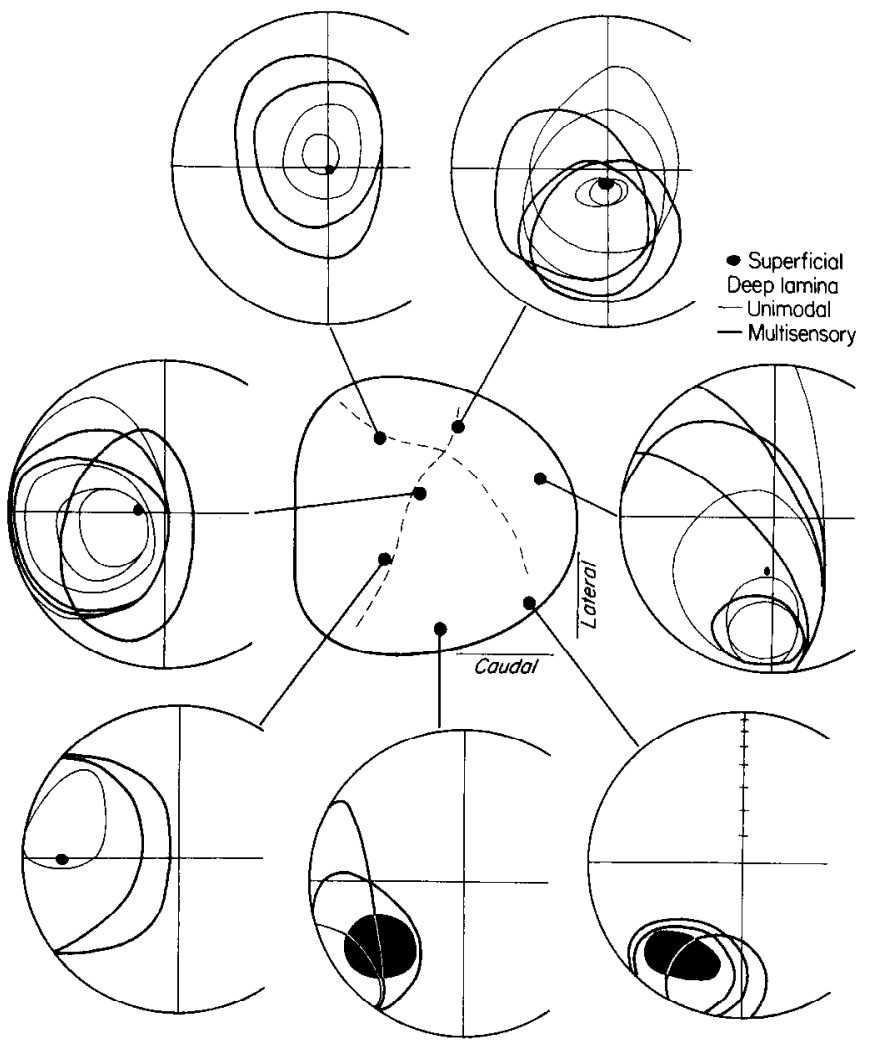

sory (heavy line) visual receptive fields located near the projection of the area centralis; more caudal recording sites yielded receptive fields located more temporal in visual space. In medial areas of the deep laminae, receptive fields generally had superior components, while more lateral recording sites had inferior components. 


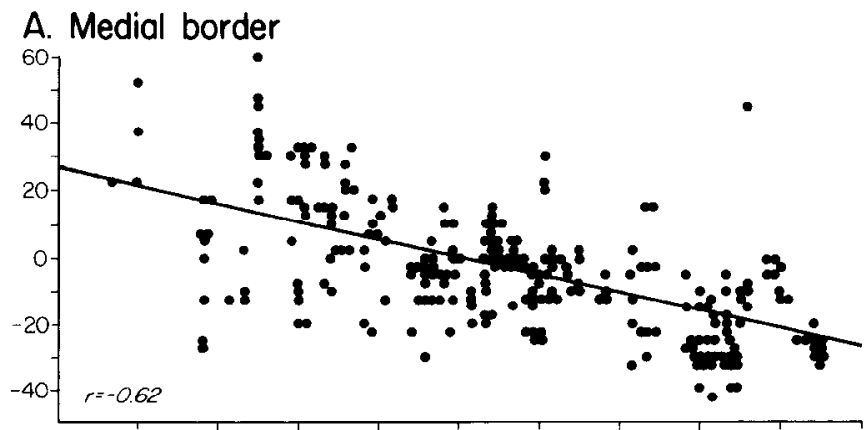

\section{Superior border}
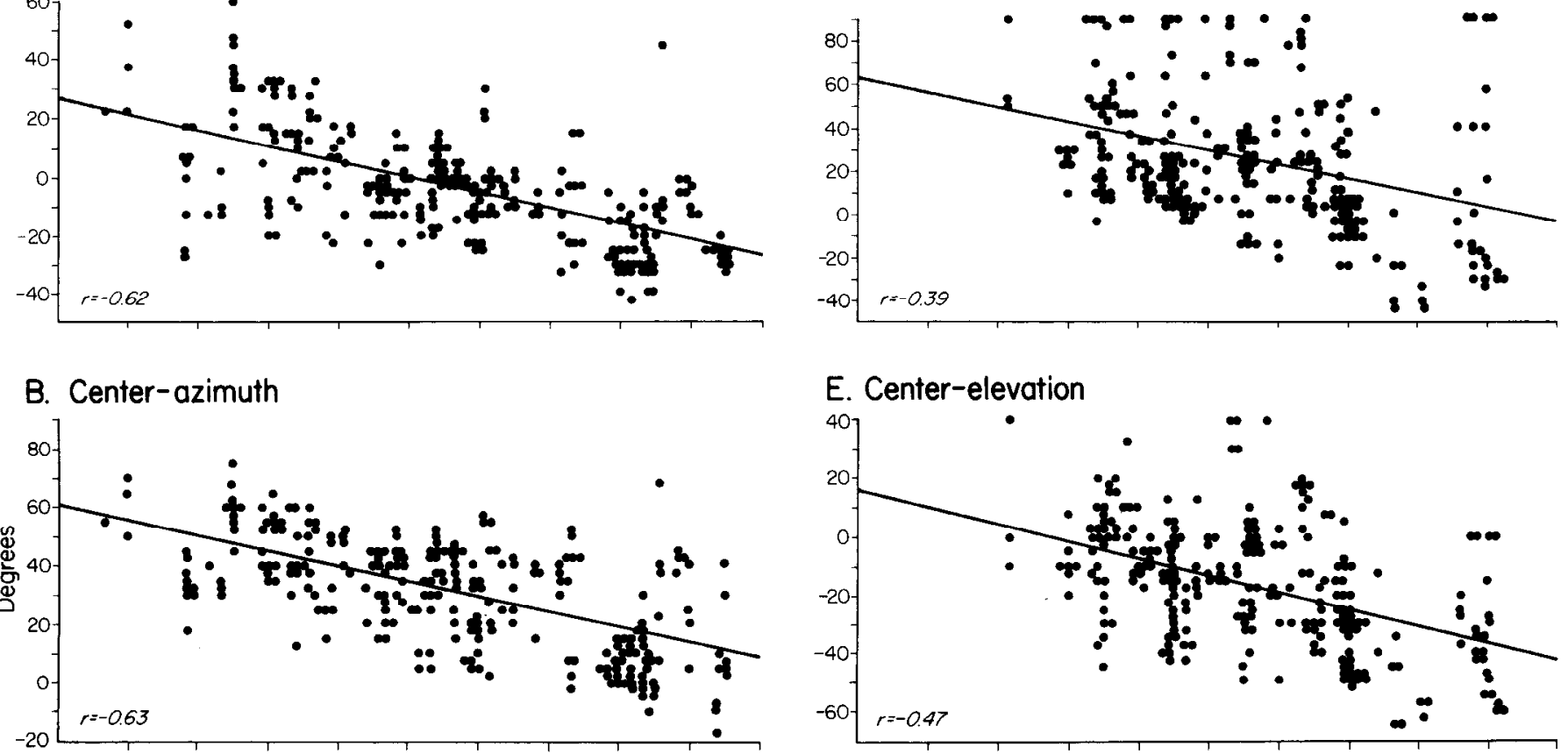

\section{Lateral border}
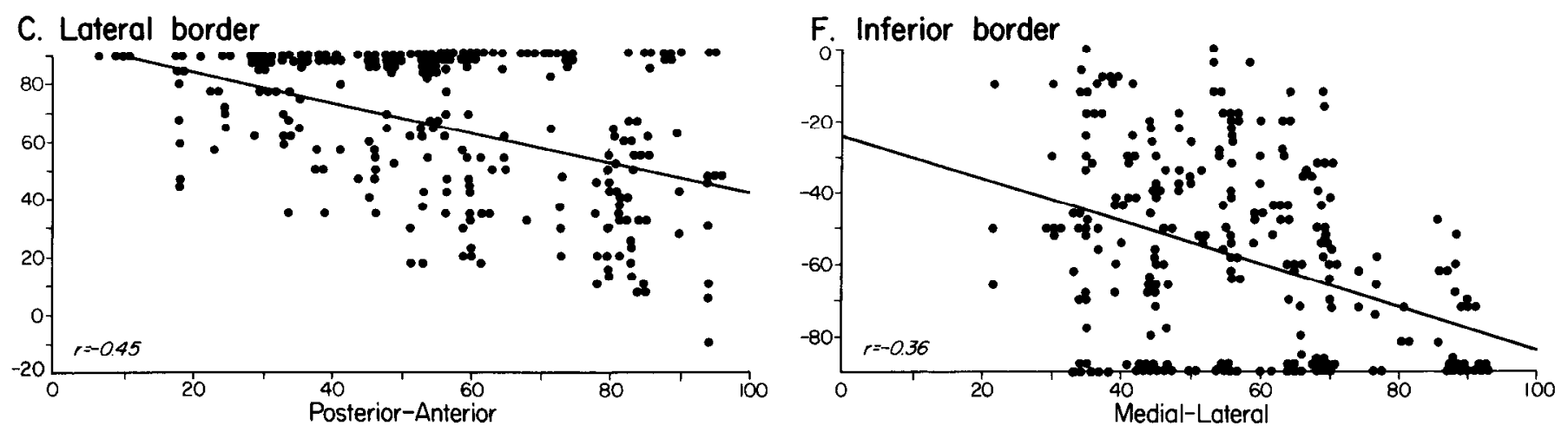

Figure 5. Relationship of receptive-field measures (border or center) to histological location of relevant cell bodies is consistent with deep-laminae visuotopy. The left column shows the medial border $(A)$, center azimuth $(B)$, and lateral border $(C)$ of the receptive field plotted against a neuron's posterior-anterior location in the superior colliculus $(0 \%=$ caudal pole, $100 \%=$ rostral pole). The graphs show a consistent and progressive temporal shift in degrees (positive numbers on $y$-axis) with more caudal (posterior) recording locations. Thus, neurons posterior in the structure tended to have temporal receptive fields. Similarly, neurons medial in the structure had superior receptive fields. The right column shows that the superior border $(D)$, center elevation $(E)$, and inferior border $(F)$ exhibit a progressive superior shift in degrees (positive numbers on $y$-axis) with more medial recording positions. Correlation coefficients $(r)$ are expressed in the lower left corner of each graph. Each dot represents a single receptive ficld.

showed the same systematic visuotopic variations with histological position.

Despite the general correspondence among receptive-field locations during a given electrode penetration, the centers of some of the decp-laminac receptive ficlds could be locatcd 30 to $40^{\circ}$ from those of others encountered in the same electrode penetration (see Fig. 6). The magnitude of this discrepancy could not be explained by the fact that vertical electrode penetrations through a curved structure will sometimes cut across radial columns and thereby produce small shifts in receptive-field positions: the data depicted in Figure $6 D$ show that receptive-field centers of superficial- and some deep-laminae cells are aligned despite the obliqueness of the recording penetration to the surface of the tissue, while the data in Figure $6 E$ represent receptivefield centers that are progressively out of register, even though the recording penetration was more closely orthogonal to the surface of the superior colliculus. Instead, a curious pattern of receptive-field shifts often appeared during individual electrode penetrations: instances of dramatic shifts $\left(>30^{\circ}\right)$ in receptivefield centers occurred in one direction, followed by a reversal of those shifts back to the original positions as still deeper neurons were sampled (see Fig. $6 A, D$ ). The presence of these eccentric points within a vertical column through the tissue, coupled with the large fields of deep-laminae neurons, would make a standard map of receptive-field centers (transformed into a series of intersecting meridians) somewhat misleading here. Such a map would not only fail to reconcile the problem of receptivefield overlap in the absence of a registry of their centers, but also would seriously underestimate the expanse of tissue to which a punctate stimulus actually has access. 

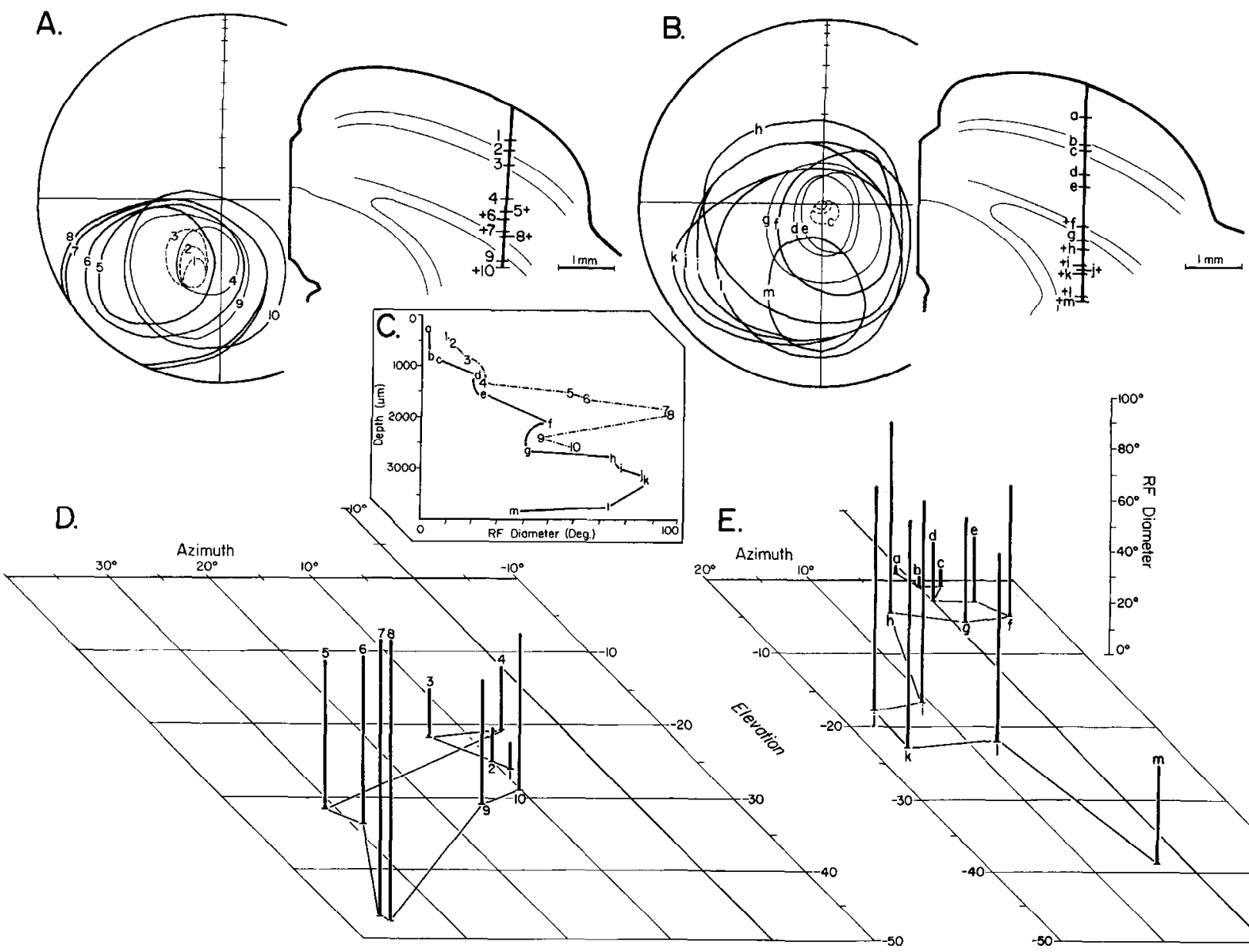

Figure 6. Within same electrode penetration, large shifts in location of receptive-field centers occurred with large increases in receptive-field size. $A$ and $B$, The receptive fields of neurons $1-10$ and $a-m$, respectively, and the histological locations of their recording sites are shown. The relationship between their receptive-field diameters and depth is depicted in $C$, and, while there is a trend toward increasing size with depth, it is not monotonic. $D$ and $E$, Schemalics of visual space ( $x$ - and $z$-axes) upon which the center of each receptive field was projected together with the size of the receptive-field diameter ( $y$-axis). Note that, as progressively deeper neurons were encountered, those with the largest receptive fields had centers most out of register with the others. See text for further description. On the histological reconstructions, pluses represent visual-multisensory neurons. All data in this figure were taken from the superior colliculus of the same animal.

\section{Deep-layer visuotopy: a population code of visual space}

Because deep-laminae visual receptive fields within the same penetration can have centers that vary dramatically in position, it was not possible to construct a visual map here by standard methods. Instead, it was necessary to determine the location (AP, ML, depth) of neurons within the deep laminae whose receptive fields shared a given point in visual space. By marking (on a representative horizontal section through the deep laminae) the location of each electrode penetration wherc at least one of the receptive fields encompassed a specified point in visual space, a region of the deep layers responsive to that visual point could be delimited. This volume of tissue representing a point in visual space has been termed a "point image" (modified from Mcllwain, 1975, 1976), and this terminology was adapted

Figure 7. Point image versus best point image in deep laminae. $A$, The location of each recording penetration $(n=64)$ is plotted on a representative horizontal section through the intermediate gray layer (all laminae ventral to the stratum opticum are collectively referred to as "deep"). Penetrations in which no receptive fields included the projec-

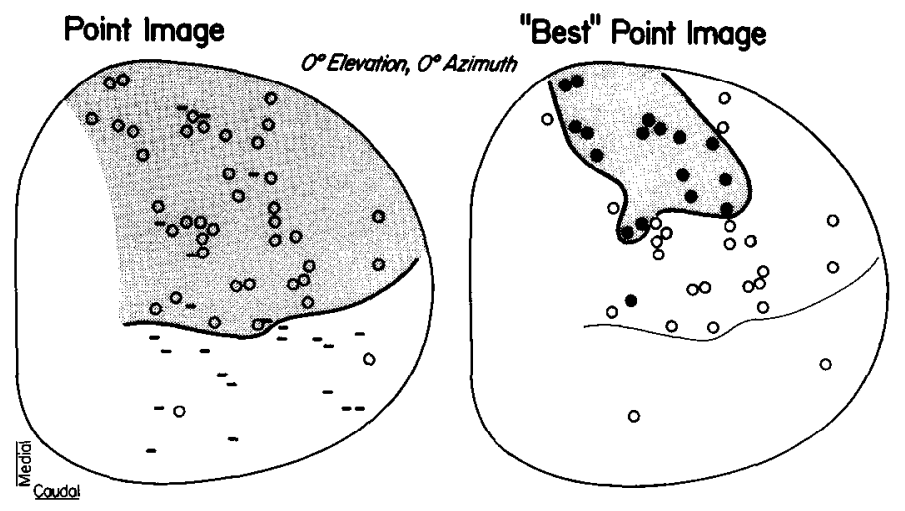

tion of the area centralis $\left(0^{\circ}\right.$ elevation and azimuth) are indicated by minuses, whereas open circles indicate those in which at least 1 receptive field encompassed this point. The stippled area includes the area of the deep laminae whose neurons had receptive fields encompassing the projection of the area centralis and constitutes its point image for that point in visual space. $B$, Replotting these data according to a more stringent criterion $(75 \%$ or greater of receptive fields per penetration indicated by solid circles) yields the more focal area of representation, or best point image, shown here. 
Figure 8. Deep-lamina visuotopy. Dots at the line intersections on the visual hemifield representation at the $t o p$ left indicate each point in the visual field whose corresponding point image in the deep layers was investigated. Each of these points in visual space is differentially represented in the deep layers, whose axes are summarized at the bottom left (constructed by overlaying best point images on the same schematic). In the center, the black areas on the schematics indicate best point images, where $75 \%$ or more of the neurons per penetration had receptive fields encompassing the specified point in the visual field, while shaded areas circumscribe the point image, indicating the locations at which at least 1 receptive field included the visual point under examination. Systematic shifts in the best point image (and point image) occurred with progressive shifts in the stimulus position, so that inferior aspects of the visual field were represented lateral (i.e., right side and bottom), superior aspects were medial and rostral (top), nasal portions were rostral-lateral (right side), and temporal regions were caudal (left side) in the superior colliculus. The schematics of the superior colliculus are traced from a horizontal section through the intermediate gray layer. Data are from 21 cats.

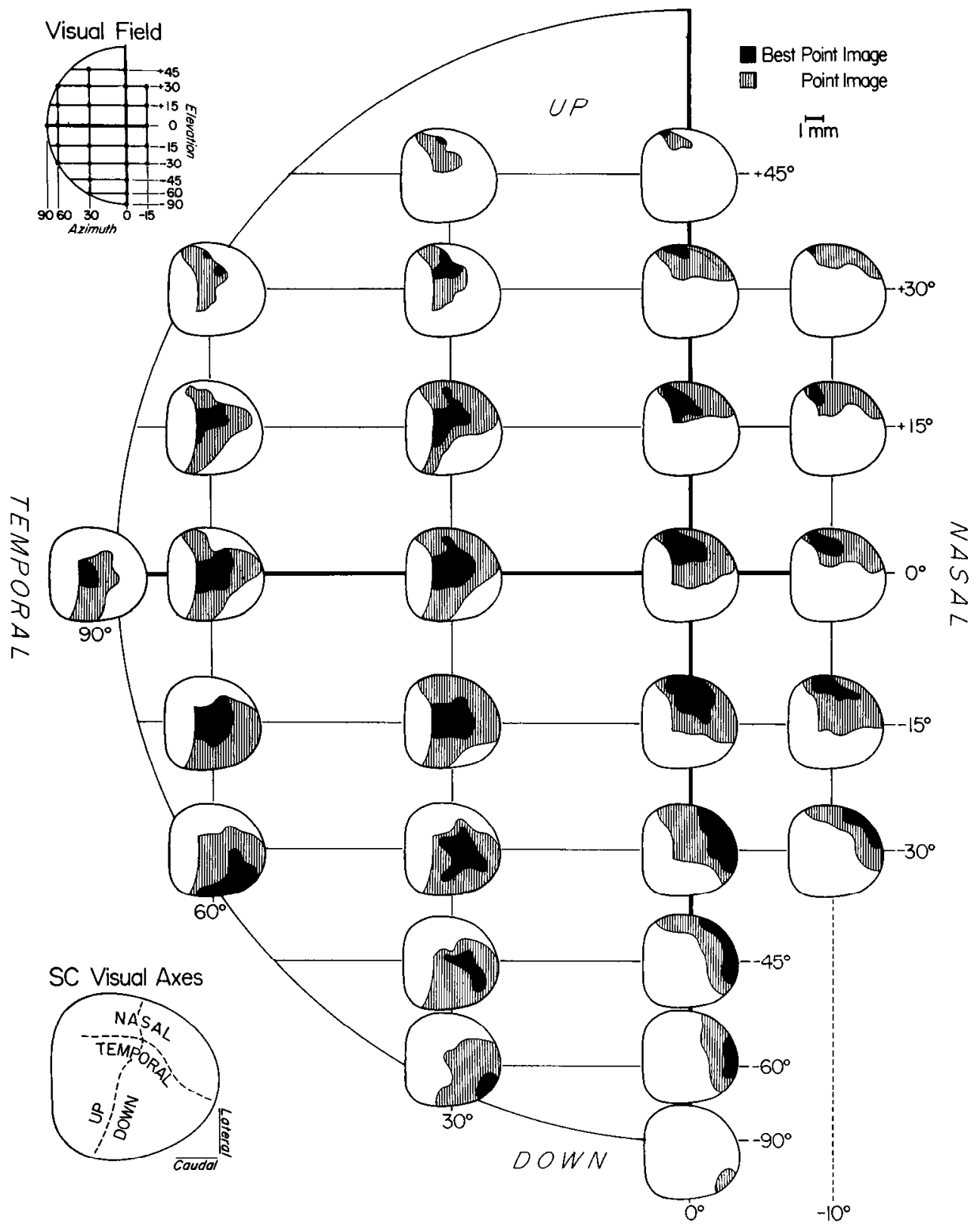

from $45^{\circ}$ superior to $-90^{\circ}$ inferior and included the azimuthal locations $90,60,30,0$, and $-10^{\circ}$. As illustrated in Figure 8 , the location of both the point image and the best point image in the superior colliculus shifted systematically with shifts in the location of the visual stimulus. The general tendency was for shifts in stimulus position from nasal to temporal in visual space to be reflected in rostral-to-caudal changes in the locations of their representation in the deep layers. However, the magnitude and angle of point image shifts were not uniform, but varied with the region of the visual field. For example, progressively shifting a stimulus from nasal to temporal along the horizontal meridian shifted the location of the best point images in the deep superior colliculus from rostral to midcolliculus. In contrast, the same nasal-to-tcmporal stimulus progression at an elevation of $-30^{\circ}$ changed the location of best point images from rostral-lateral to the caudal pole. Similarly, the general

trend for the change in best-point-image location with shifts in for a series of points that crisscrossed the visual field. These points in visual space were spaced at $15^{\circ}$ intervals in elevation here as well. As can be seen from Figure $7 A$, the point image point is not of equal strength throughout the point image, beified point, while at other positions, only a few receptive fields met the same criterion. To determine the subregion of a point image that best focused the representation of a given point in the visual field, the data were replotted to segregate those locations where $75 \%$ or more of the receptive fields shared the specified point (called the "best point image"), as shown in Figure $7 B$.

To examine the visuotopy within the decp laminac, point images and best point images were systematically determined 


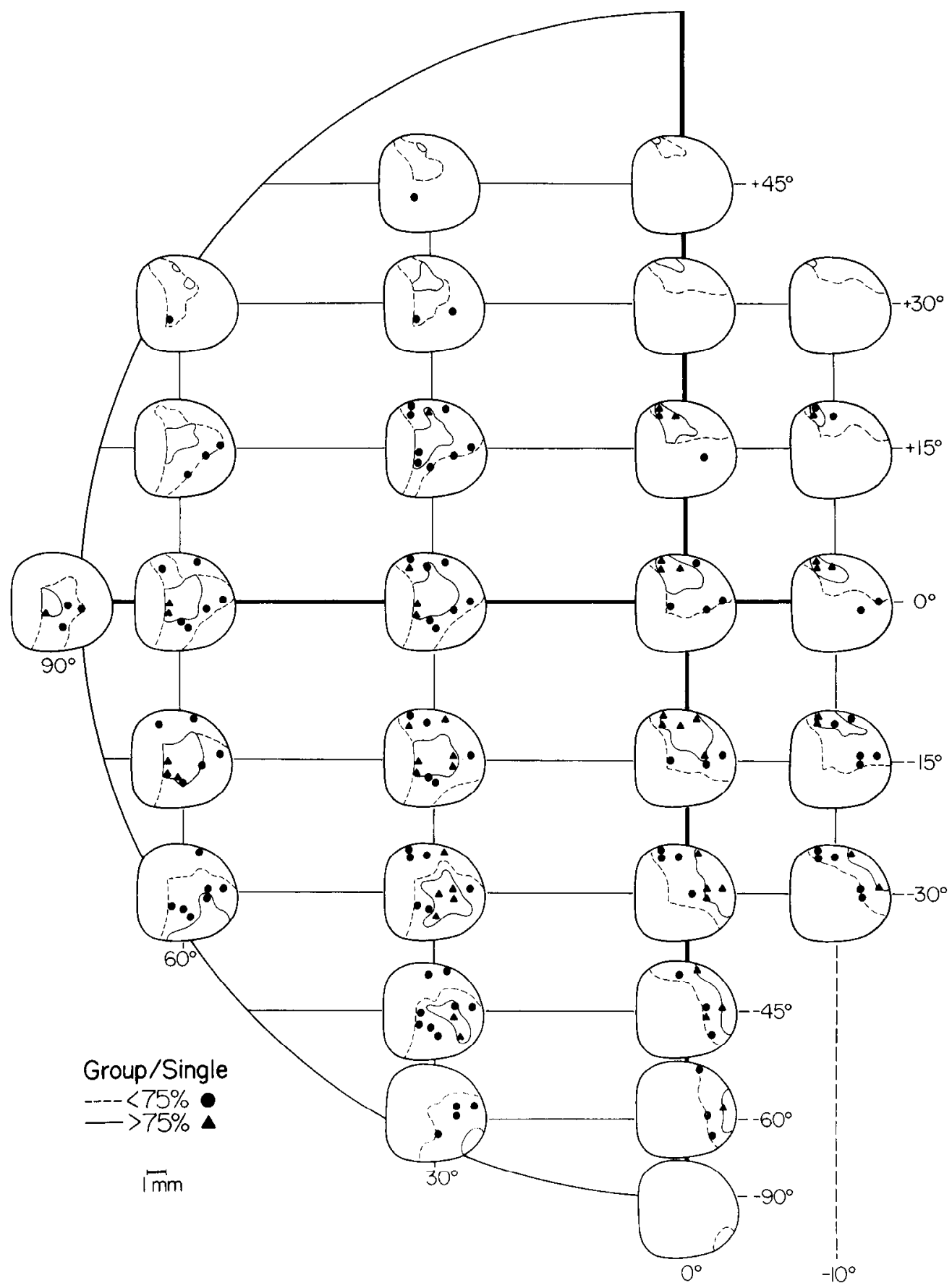

Figure 9. Deep-lamina visuotopy in single animal reflects that established by data pooled from entire group of animals. In the schematics shown here, the solid lines are tracings of the best point images from Figure 6 , and the dashed lines are the point images from the same figure. Each triangle represents a penetration in this particular animal in which $75 \%$ or greater of the neurons per penetration had receptive fields that encompassed a specific point in visual space, while the circles indicate penetrations in the same animal in which at least 1 but $<75 \%$ of the receptive fields included the same visual point. Note the close correspondence among the most highly effective penetrations (triangles) and of all effective penetrations (triangles and circles) in this single case to the distribution of best point images and point images from the population of animals studied and summarized in Figure 8. the vertical position of the stimuli were along the mediolateral axis of the structure. However, the shifts of point images were more dramatic for representations of nasal as compared to temporal stimuli. It should be noted, however, that, because of the inaccessibility of a small strip of medial tissue obscured by the sagittal venous sinus, the area of the deep layers representing superior visual space may be underestimated here.

To determine if visuotopic variability among animals might have exaggerated the size of a given point image, the pooled data of Figure 8 were compared directly to the data generated in 4 well-studied individual cases. One of the individual-group comparisons is illustrated in Figure 9 and was constructed by plotting the data from that animal (the most widely spaced electrode penetrations were made in this case) directly onto the group representation. As can be seen, $88 \%$ of the data points from the individual experiment fell inside their predicted bestpoint-image locations on the composite map, and $90 \%$ fell within the group point image. The range of individual-group coincidence among all animals compared was from 84 to $90 \%$ for best point images, and from 79 to $99 \%$ for point images.

The manner in which positions of visual elevation and azimuth are represented in the deep layers of the superior colliculus are summarized in Figure 10. Even using the most stringent criterion applied here (i.e., $75 \%$ or more of the neurons in an electrode penetration included the same point in the visual field) to construct a map of azimuth and elevation did not eliminate 


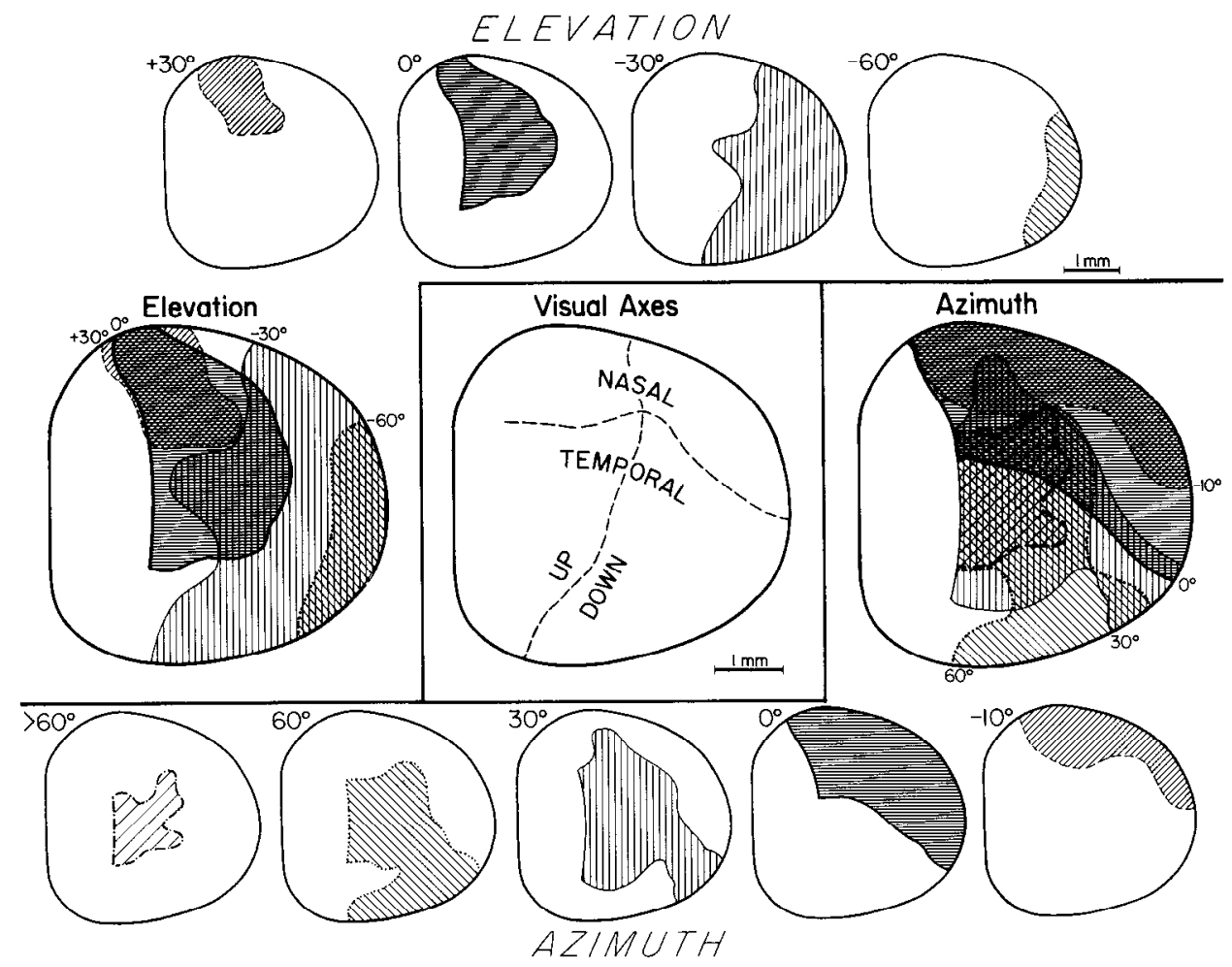

Figure 10. Summary of pooled data showing zones of maximal representation, or best point images, representing levels of elevation (left and $t o p$ ) or azimuth (right and bottom) of visual field. Large zones represent different levels of elevation and azimuth, and there is considerable overlap among adjacent zones. Nevertheless, as summarized in the central schematic, there is a definite progression of superior-to-inferior (elevation) visual space from the rostromedial to the caudolateral aspect of the superior colliculus and of nasal-to-temporal (azimuth) visual space along the anterior-to-posterior medial axis of the structure.

the substantial overlap that undoubtedly reflects the large receptive fields of deep-laminae neurons. Nevertheless, this figure shows that nasal-to-temporal visual space is generally represented in a rostral-to-caudal fashion in the deep layers, while superior-to-inferior visual space is represented from rostral-medial to caudal-lateral in the superior colliculus.
Within this topography, there is an expansion of central visual space, and any point that fell within $30^{\circ}$ of the projection of the area centralis had access to an average of $12.6 \%( \pm 7.7)$ of the deep superior colliculus area. Curiously, points lateral or inferior to the visual axis (e.g., $0^{\circ}$ elevation, $30^{\circ}$ azimuth $=23.1 \% ;-15^{\circ}$ elevation, $30^{\circ}$ azimuth $=20.1 \% ;-15^{\circ}$ elevation, $0^{\circ}$ azimuth $=$
Figure 11. Expanded representation of central and near-central visual space in deep superior colliculus. The area $(y$ axis) of each point image within the deep layers is plotted at its corresponding location in visual space. Note that the point images representing central and inferior points occupy far greater proportions of the tissue than those representing points in more peripheral, superior, or ipsilateral visual space.

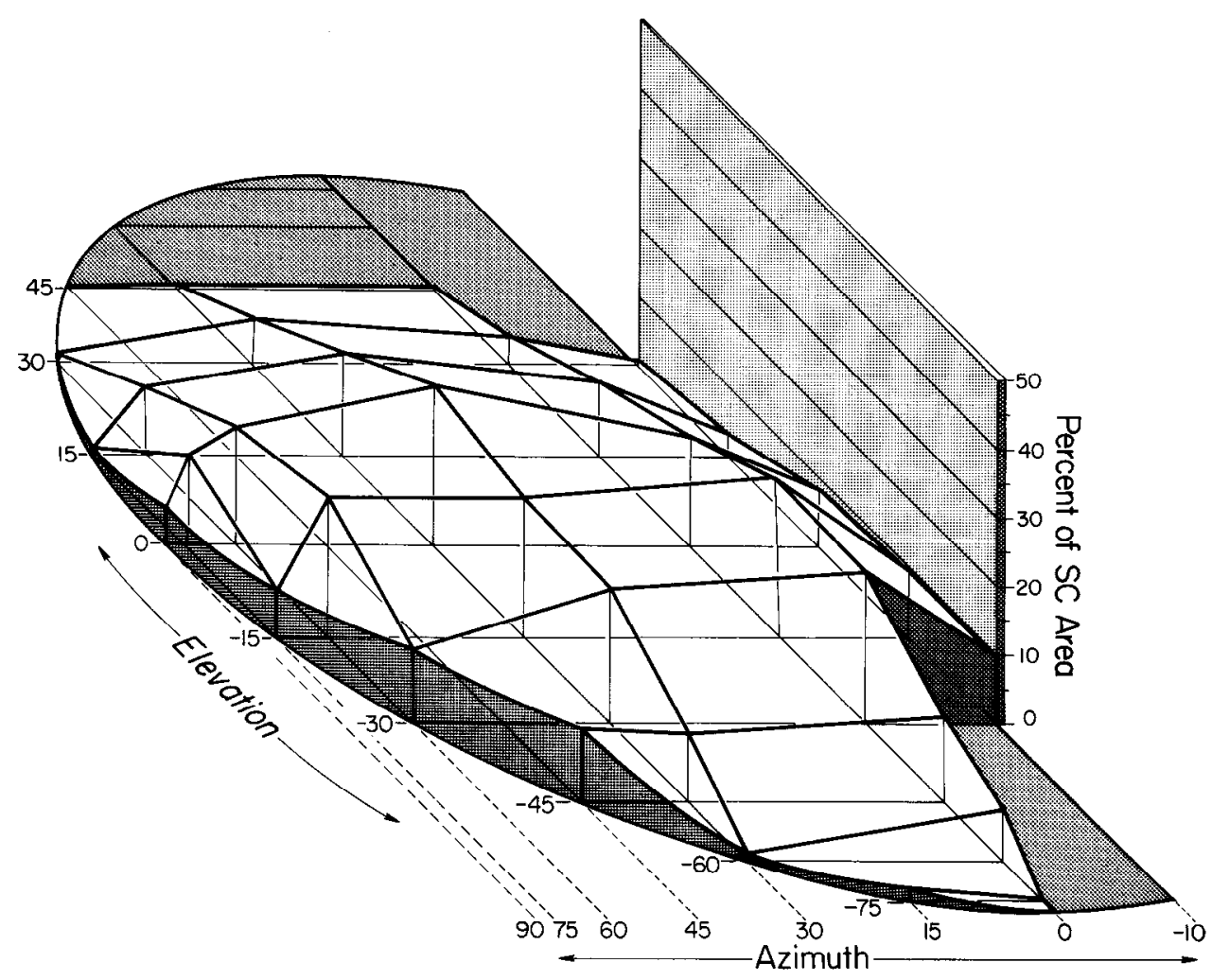




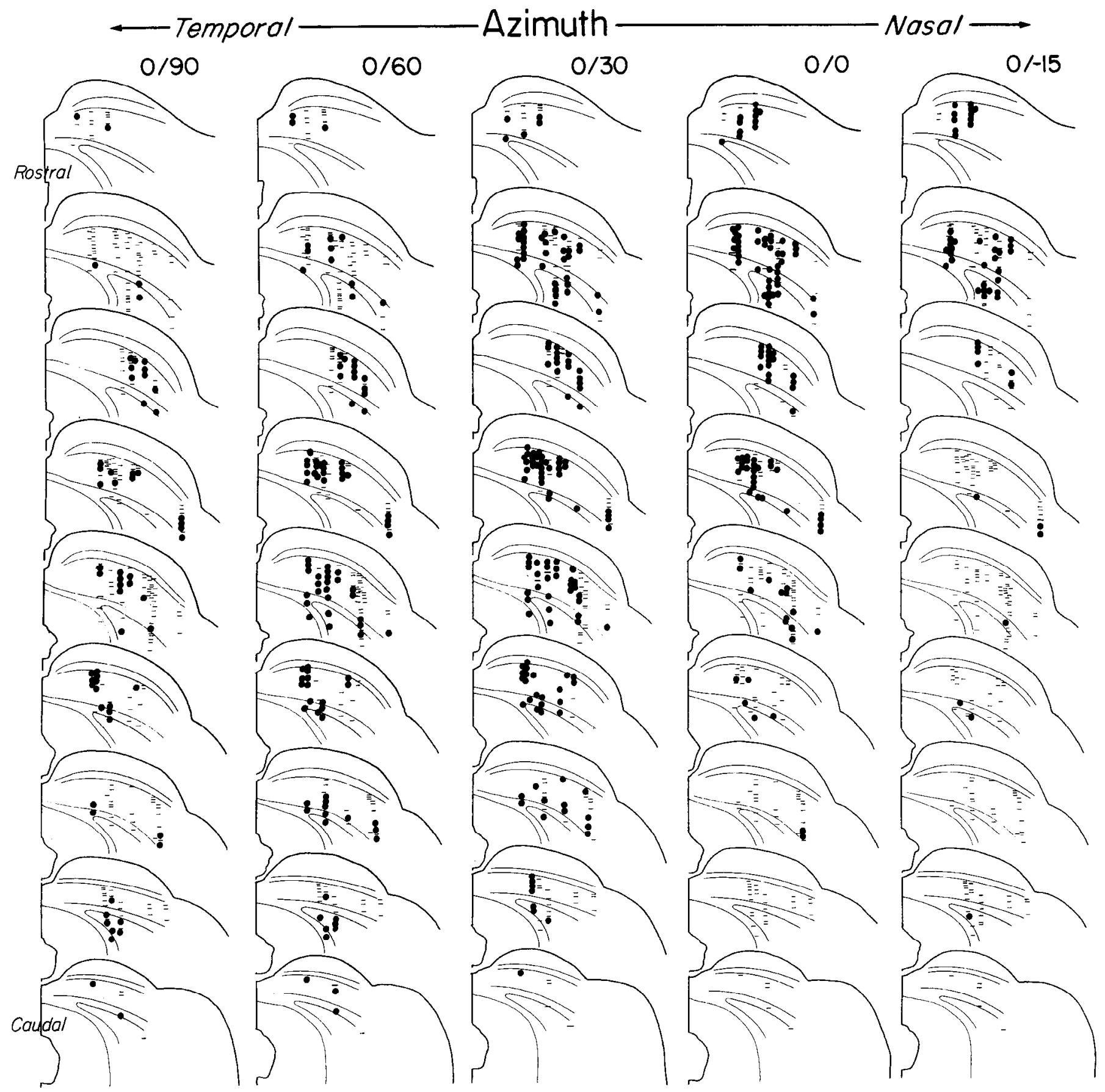

Figure 12. Deep-layer visuotopy extends through depth of deeper laminae. Coronal sections taken at regular intervals (10\% or approximately 450 $\mu \mathrm{m}$ ) through the superior colliculus are arranged from rostral (top) to caudal (bottom). Dots represent neurons whose receptive fields include the location of the visual point specified at the top of the column of sections; dashes indicate that the visual point was not contained within the neuron's receptive field. Each column of sections and neurons is identical, except that the differential distribution of dots indicates the shift in point image location according to the change stimulus position.

$23.1 \% ;-30^{\circ}$ elevation, $0^{\circ}$ azimuth $\left.=22.1 \%\right)$ had the largest best-point-image areas of all. With these best point images pooled together, the representation of the central $30^{\circ}$ of visual space occupies nearly $3 / 4(72.7 \%)$ of the deep-laminae area. In contrast, the other best point images that subserve points $45^{\circ}$ eccentric or greater in the visual field averaged only $7.1 \%( \pm 6.2)$ of the area of the deep layers. Collectively, these "peripheral" best point images encompassed a total of $60.5 \%$ of the deep superior colliculus and shared some of the same regions representing more centrally located stimuli. The percentage of the area of the deep laminae occupied by each best point image is depicted in Figure 11, where these central-peripheral distinctions are evident.

To examine the access of stimuli at different points in visual space to neurons along the vertical extent (depth) of the superior colliculus, the data for each point image shown in Figure 8 was replotted on coronal sections at $10 \%$ intervals along the rostrocaudal axis of the superior colliculus. As illustrated in Figure 


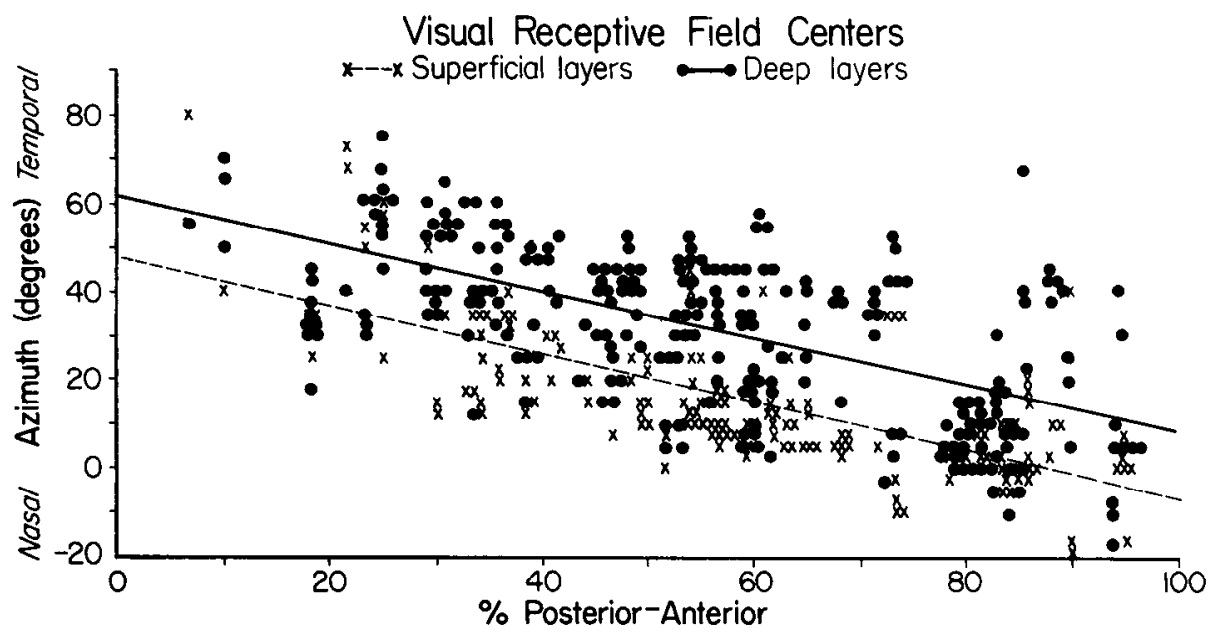

Figure 13. Superficial- and deep-laminae maps share similar vertical and horizntal axes. Plotted here are the azimuth (top) and elevation (bottom) of receptive-field centers versus the posterior-anterior or medial-lateral location, respectively, of neurons within the superficial $(X s)$ or deep (solid circles) subdivisions of the superior colliculus. These plots reveal nearly the same systematic shifts in receptive-field position with laterality or posterior-anterior location in the structure for both superficial- and deep-layer neurons. The slopes of these relationships are not significantly different from one another, but there are substantial differences in their intercepts.

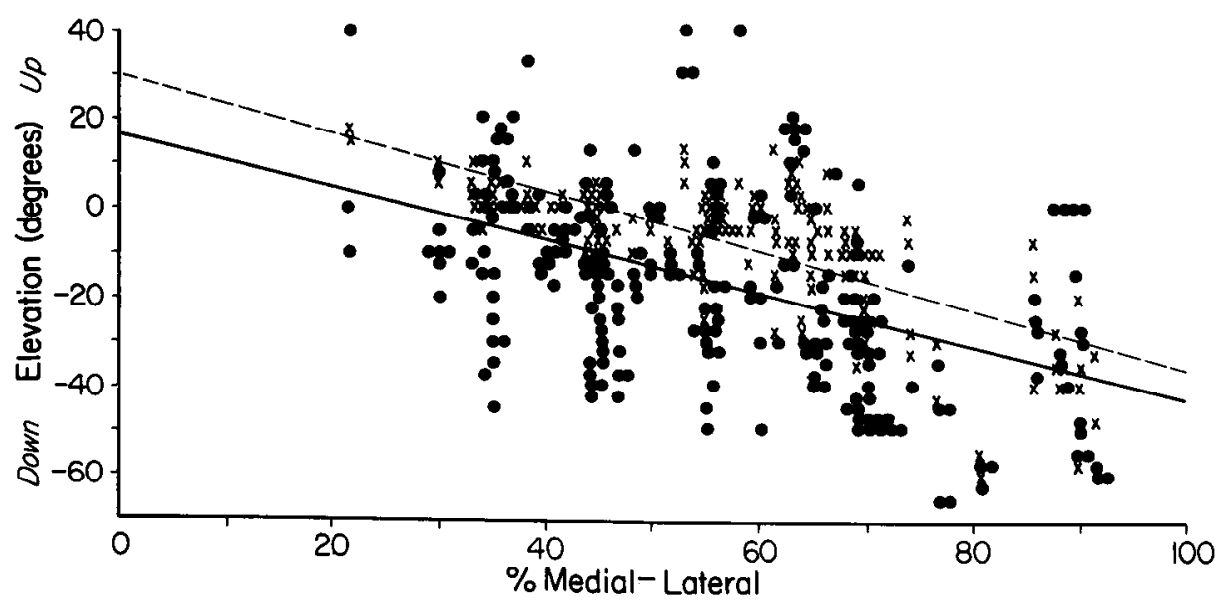

12 , each point image was primarily constituted by neurons in the intermediate gray and white layers, with proportionally fewer neurons from greater depths involved. In no case was the representation of a point in the visual field found exclusively within a specific lamina.

\section{Superficial-deep-laminae visuotopic registry}

The well-known superficial-layer visual map (Feldon et al., 1970) was constructed in a manner substantially different from that used here to examine the deep-laminae visual representation, making direct comparisons difficult. Nevertheless, data gathered from the superficial layers during the current investigation were similar to what has been presented previously and were used to compare the superficial- and deep-laminae visuotopies directly. The relationships of superficial-layer receptive-field measures (superior, inferior, lateral, and medial borders, center azimuth, and center elevation) to the AP or ML location of the neuron showed similar trends to what has already been observed for deep-layer receptive fields. In addition, when the center azimuth and center elevations were plotted against their AP and ML positions for both superficial- and deep-layer neurons, the result was the pairs of nearly parallel regression lines illustrated in Figure 13. The similarities in their slopes reflect the fact that the 2 maps share similar axes. However, the intercepts for the superficial- and deep-laminae receptive fields were substantially different, and this was most evident for azimuth (center azimuth vs. AP position, $p=0.002$; center elevation vs. ML position, $p$ $=0.41$; analysis of covariance). These differences may be due to the general expansion, in inferior and lateral directions, of many of the deep-layer receptive fields relative to their more superficial counterparts. For example, whereas the superficiallayer map extended from approximately $-20^{\circ}$ in ipsilateral space to $70^{\circ}$ temporal and $25^{\circ}$ superior to $-50^{\circ}$ inferior, the deeperlaminae representation was far more extensive. It included the entire contralateral visual field (from 0 to $>90^{\circ}$ temporal and from $90^{\circ}$ superior to $90^{\circ}$ inferior) and extended to $-40^{\circ}$ in ipsilateral space.

Superficial- and deep-layer neurons encountered within the same electrode penetration very often had receptive-field centers in substantially different locations (see, e.g., Fig. 6), and a general but systematic inferior and lateral shift was noted, as shown in Figure $14, A$ and $B$. This effect was most obvious with the more eccentrically positioned receptive fields (e.g., $\left.>15^{\circ}\right)$. Conversely, centrally placed receptive fields in the superficial and deep layers showed a greater degree of alignment.

\section{Deep-layer unimodal versus multisensory visuotopy}

In the deep laminae, multisensory visual neurons outnumbered unimodal visual neurons in the sample obtained here (71 vs. $29 \%$ ), and this is in agreement with our previous findings (see 
Meredith and Stein, 1986b). Furthermore, the proportion of visual-multisensory neurons representing each point of visual space examined was greater than the visual-unimodal neurons at all locations in the visual field. The difference in these proportions grew from 59 to $69 \%$ for representations near the projection of the visual axis and became progressively greater at increasingly peripheral locations, reaching a maximum (93-95\%) at positions of $75^{\circ}$ azimuth or greater (Fig. 15).

\section{Discussion}

These experiments have demonstrated the presence and organization of a visual map in the deeper laminae of the cat superior colliculus. This visuotopy includes nearly all aspects of the contralateral visual field, as previously documented with behavioral perimetry methods (see Sprague, 1966; Sherman, 1974; Hardy and Stein, 1988). It extends from approximately $40^{\circ}$ nasal to the vertical meridian to more than $90^{\circ}$ temporal to it, and from $90^{\circ}$ inferior to the horizontal meridian to $90^{\circ}$ superior to it. The map exhibits a tilt with relation to the anatomical axes of the superior colliculus, so that the vertical meridian runs from rostromedial to lateral, while the horizontal meridian runs obliquely from the rostral pole toward the caudomedial border. As is true of most other visuotopies, the map is not a simple linear transformation of visual space, but exhibits a considerable expansion of central and inferior visual space and a concurrent compression of far temporal space.

Although this deep-layer visual map does have a number of features in common with the well-known superficial-layer representation (e.g., see Feldon et al., 1970), and these are discussed below, it is distinct by virtue of the magnitude of its constituent receptive fields. Deep-laminae receptive fields have diameters that average $66.9^{\circ}$ (range, $13.5-150.5^{\circ}$ ), a considerable increment over those found in the superficial layers and much larger than those of its input neurons from retinal $\mathrm{Y}$ cells (mean, $<2^{\circ}$ diameter), lateral suprasylvian (mean, 14-16 ), and anterior ectosylvian (mean, $15-30^{\circ}$ ) cortical neurons (Hubel and Wiesel, 1969; Spear and Baumann, 1975; Rusoff and Dubin, 1977; Marzi et al., 1982; Mucke et al., 1982; Olson and Graybiel, 1987). This suggests that, like their nonvisual counterparts, deep-laminae visual receptive fields are constructed by the convergence of spatially related but not coincident receptive fields (Clemo and Stein, 1984, 1986; Meredith and Clemo, 1989). Given the likelihood of extensive convergence onto neurons with expansive dendritic trees (up to $1400 \mu \mathrm{m}$ in diameter; Moschovakis and Karabelas, 1985), the fact that deep-layer visual receptive fields are extremely large is not surprising. One consequence of these large receptive fields is that a specific point in visual space has access to a large block of deep-layer tissue, an arrangement consistent with the hypothesis that populations of neurons are necessary to code for stimulus location (Capuano and McIlwain, 1981) and for targeting saccadic eye movements (Van Gisbergen et al., 1987; Lee et al., 1988).

The functional significance of large deep-laminae receptive fields has been a subject of speculation. One reasonable suggestion is that a very large number of deep-laminae neurons are likely to be activated by a given visual stimulus, thereby increasing the probability of evoking colliculus-mediated behavioral responses (Stein et al., 1976). This may only be a partial explanation, however, because the majority of these deep-layer "visual" neurons also are responsive to nonvisual (auditory and/ or somatosensory) stimuli, and the extensive nature of the visual (and nonvisual) receptive fields helps to maintain their topo-

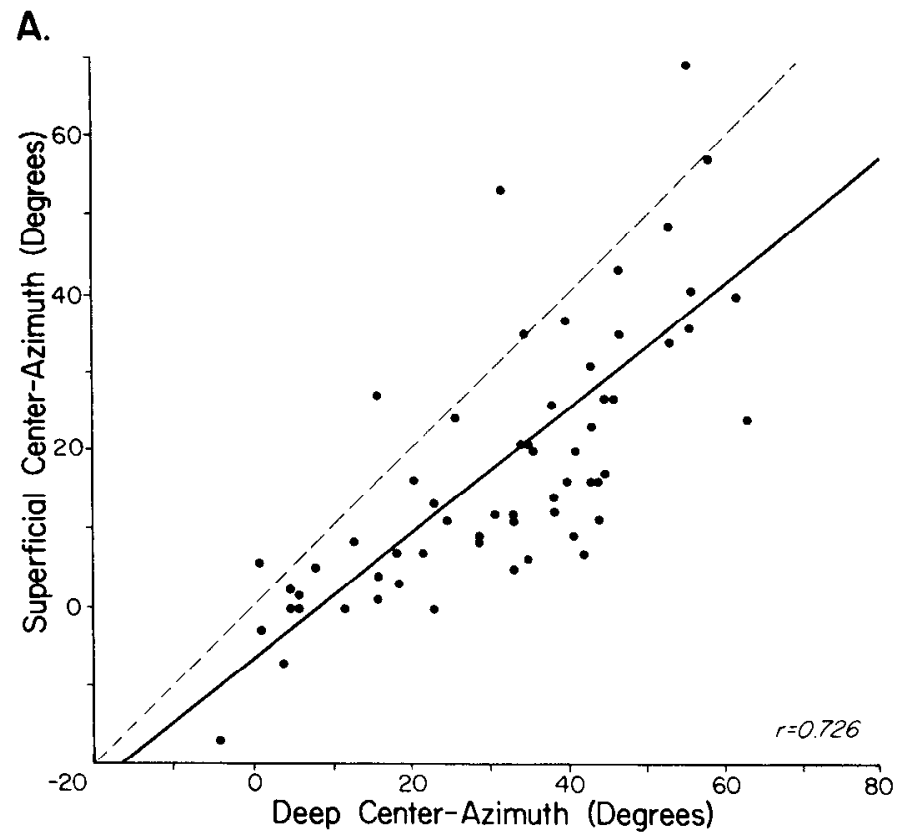

B.

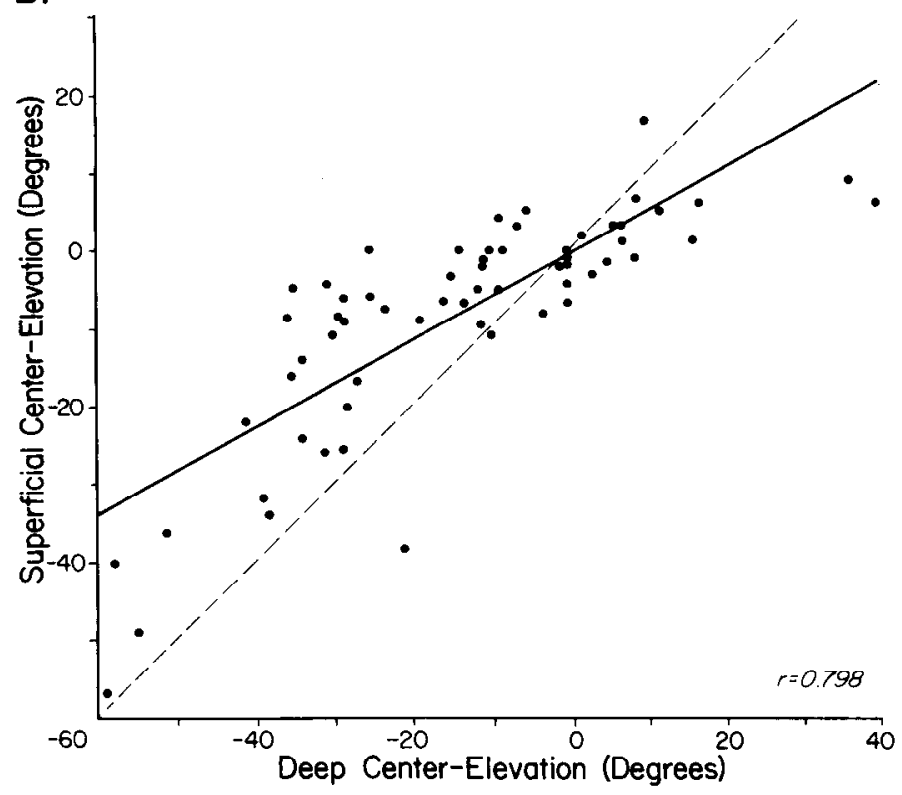

Figure 14. Relationship of superficial- and deep-layer receptive fields within same penetration. Plotted here are the mean values for azimuth $(A)$ and elevation $(B)$ of receptive-field centers of superficial-layer neurons versus those for deep-layer neurons in the same penetration. The dashed lines represent a correlation of unity, where variation in the superficial-layer receptive fields would be exactly paralleled by those of the underlying deep-laminae receptive fields. The solid lines show the actual relationship obtained. Note that this comparison reveals that average receptive-field centers, within the same penetration, tend to show a general lateral $(A)$ and inferior $(B)$ drift as recording progresses from the superficial to deep layers, especially for the more eccentrically located receptive fields.

graphic registry despite minor shifts of the peripheral sensory organs (i.e., receptive-field overlap is easier to maintain between 2 large receptive fields than 2 small ones). The topographic register among receptive fields of different modalities within the same multisensory neuron is essential for multisensory integration, a major role of deep-layer neurons (Meredith and Stein, 
Figure 15. Point images in deep layers of superior colliculus are dominated by multisensory-visual neurons. For each point image in the grid representing points in visual space (see Fig. 8, top left), 59 to $95 \%$ of the neurons received inputs from other sensory modalities (visual-multisensory: upper contour grid), and these proportions tended to increase with increasing eccentricity. Consequently, the deep-laminae map of visual space is dominated by multisensory neurons.

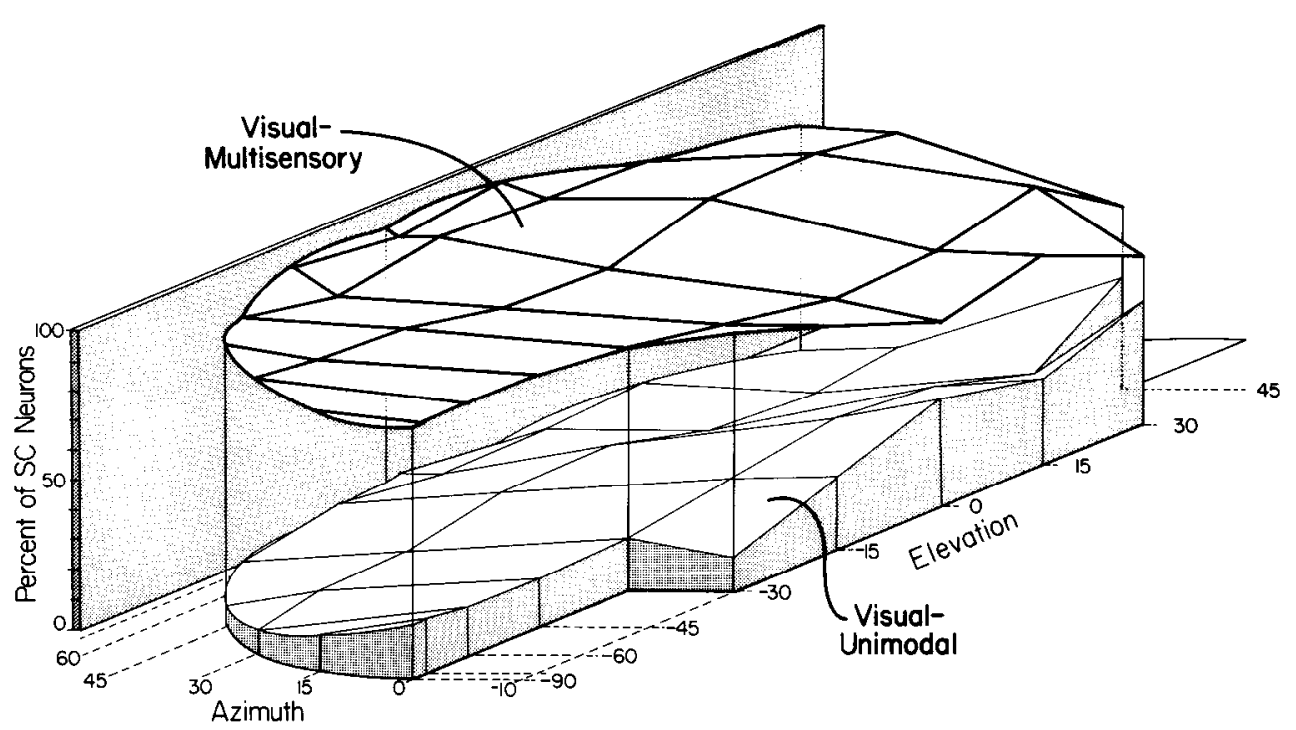

1986a,b). This suggestion leads one to expect that organisms less able to move their visual and nonvisual sensory organs independently (and thereby misalign them and their respective receptive fields) would exhibit relatively smaller receptive fields. Such appears to be the case in visual-auditory ncurons in the barn owl tectum, an animal incapable of significant independent displacement of its eyes or external auditory apparatus (Knudsen, 1982). On the other hand, considerably larger receptive fields have been found in the deep layers of the cat and ferret superior colliculus (Gordon, 1973; King and Palmer, 1983; Middlebrooks and Knudsen, 1984; Meredith and Stein, 1986a,b), and these animals exhibit a wide range of independent eye and pinnae movements.

The fact that the vast majority of visually responsive neurons also receive nonvisual inputs underscores the multisensory nature of the visual representation in the deep layers of the superior colliculus. Because it is well established that visual activity evoked in these neurons can be influenced dramatically by stimuli from other modalities (Meredith and Stein, 1983, 1986a,b), it might not be entirely accurate to think of this representation as equivalent to visual maps in purely visual areas of the brain, including the superficial layers of the same structure (see below). Instead, the deep layers may best be regarded as a topographically organized multisensory representation whose circuitry can be accessed through individual or combined sensory channels. In this light, then, the developmental realignments of one sensory map in the superior colliculus that result from neonatal manipulations (e.g., sensory experience or surgical intervention) of the map of another modality (Knudsen and Knudsen, 1985; Knudsen, 1987; King et al., 1988) may depend on both modalities converging on the same neurons. Only then can their inputs be matched and selected on the basis of activity-dependent interactions. One might then predict that, unless the topographic arrangement developed by multisensory neurons can extend (by some as yet unknown means) to their unimodal neighbors, these unimodal neurons (of the modality not directly manipulated) may show little or no realignment and exhibit an "aberrant" topography, a hypothesis that can be readily tested.

\section{Superficial-deep-laminae relationship}

It is well established that the different visual representations found in the superficial and deep laminae share a variety of fcaturcs. Visual ncurons in both subdivisions show similar preferences for stimulus movement, velocity sensitivity, and direction selectivity, as well as exhibit homogeneously organized receptive fields (for review, see Stein, 1984). Furthermore, the maps in both regions share similar visuotopic axes and exhibit expansions of the representations of the central and inferior visual field. Although it is certainly possible that mechanical factors during development encourage similar distributions of visual afferents and thereby produce parallel maps, the existence of these organizational and receptive-field commonalities (see Ogasawara et al., 1984) and the presence of some superficialdeep-laminae interconnections (Behan and Appell, 1987; Mooney et al., 1988a,b; Moschovakis et al., 1988; Rhoades et al., 1989) argue for some exchange of information. Current evidence, however, indicates that visual processing in the deep layers is independent of superficial-layer function (Ogasawara et al., 1984), suggesting that the interconnections between the laminae might not carry information critical for the visual functions usually assessed experimentally.

Consistent with the idea that the superficial visual map is largely unrelated to deep-laminae function is the observation that superficial-laminae lesions do not produce the visually guided deficits that are induced by lesions that encroach on deep laminae (Casagrande and Diamond, 1974). Furthermore, the 2 divisions of the structure depend on different sources for their visual properties. Superficial- but not deep-laminae neurons receive their visual inputs primarily via a robust retinotectal projection (Graybiel, 1975; Harting and Guillery, 1976) and a dense corticotectal projection from the striate cortex (Kawamura et al., 1974; Gilbert and Kelly, 1975). Removal or temporary deactivation of the striate corticotectal projection profoundly affects the characteristics and responsiveness of superficial-laminae visual neurons (Wickelgren and Sterling, 1969; Stein and Arigbede, 1972; Berman and Cynader, 1975; Stein and Magal- 
haes-Castro, 1975; Stein, 1978; Hardy and Stein, 1988), but has little demonstrable effect on deep-laminae visual neurons (Ogasawara et al., 1984). On the other hand, deep-laminae visual neurons receive their inputs largely from projections outside the "primary" thalamocortical system, much like their nonvisual counterparts (Stein et al., 1983; Clemo and Stein, 1984, 1986; Meredith and Clemo, 1989). Only a sparse retinotectal and striate corticotectal projection reaches deep-laminae neurons (Berson and McIlwain, 1982; Beckstead and Frankfurter, 1983), but of critical importance is their "extrastriate" input from the lateral suprasylvian cortex (Ogasawa et al., 1984; Hardy and Stein, 1988) and the ectosylvian visual area (Mucke et al., 1982).

In terms of their visuotopic maps, clear differences between superficial and deep laminae are apparent. One of their most notable contrasts is in the size of the constituent receptive fields and, thus, the resolution of the maps. The comparatively small visual receptive fields of superficial-layer visual neurons and their well-ordered arrangment is not matched by the rather large deep-laminae auditory (Middlebrooks and Knudsen, 1984; Meredith and Stein, 1986a) and somatosensory receptive fields (Stein et al., 1976; Meredith et al., 1990) despite their general topographic correspondence. Nevertheless, because the superficial visual map and the deep-laminae nonvisual representations share similar axes, exhibit similar magnifications of different regions of the sensory world (e.g., central and inferior sensory space; see Stein et al., 1976), and were thought to be functionally related, a good deal was made of their approximate topographic register (Drager and Hubel, 1975; Stein et al., 1976; Stein and Dixon, 1978). Indeed, the present study offers direct evidence that superficial and deep visual representations share similar axes. Yet, close alignment of the superficial and deep visual maps appears to occur only in the more rostral aspects of the superior colliculus. Furthermore, the deep layers contain a substantially more extensive representation of the visual field than is found superficially. Thus, while representations of visual space appear to be most closely aligned centrally, the deep layers represent more peripheral regions in the same area of tissue than are found superficially, thereby shifting the maps (especially their peripheral aspects) relative to one another (a situation reminiscent of the relationships of the deep-infrared and superficial-visual maps found in the rattlesnake tectum; see Hartline, 1984).

It is important to emphasize that the organization of the deeplayer visual map described here, as well as its relationship with that in the superficial layers, relies on general trends derived from populations of receptive fields. While the receptive fields of deep-layer neurons frequently overlapped the same area of visual space occupied by receptive fields of their more superficial neighbors within the same elctrode penetration (thereby providing the coarse superficial- to deep-laminae topographic register), the centers of superficial-layer receptive fields were unreliable indicators of individual deep-layer-neuron receptivefield centers. These observations are similar to those of McIlwain (1976), who asserted that, "as receptive field diameter increases, the location of the receptive field center becomes a poorer estimator of the retin[otopic] origin of afferent fibers." Consequently, estimates of the likelihood or direction of projection lines from superficial to deep layers are difficult to make with the data presented herc (but see Rhoades ct al., 1989). Given the large and variable size of deep-layer visual receptive fields, the weak visuotopic relationships observed between individual receptive-field centers from the same (or even adjacent) electrode penetrations were not unexpected. However, when the receptive fields of populations of neurons were assembled according to visual-field commonalities, a more precise visuotopy became apparent (see also McIlwain, 1975; Capuano and Mcllwain, 1981).

\section{Conclusions}

These data indicate that the visually responsive neurons of the deeper layers of the superior colliculus are arranged in a visuotopic map of the contralateral (and a portion of the ipsilateral) visual field. Although this representation has axes similar to those found in the well-known superficial-layer map, its nonvisual inputs and large receptive-field sizes clearly distinguish it from its more dorsal neighbor. The properties of the deeplaminae visual representation closely match those of the nonvisual representation located here and are best considered as a component of a cohesive, multisensory, functional unit that plays a critical role in effecting behavioral responses to a wide variety of sensory stimuli.

\section{References}

Apter J (1945) Projection of the retina on the superior colliculus of cats. J Neurophysiol 8:123-134.

Beckstead RM, Frankfurter A (1983) A direct projection from the retina to the intermediate gray layer of the superior colliculus demonstrated by anterograde transport of horseradish peroxidase in monkey, cat and rat. Exp Brain Res 52:261-268.

Behan M, Appell PP (1987) Local projections of neurons in the deep layers of the cat superior colliculus: a study using Phaseolus vulgaris leucoagglutinin (PHAL-L). Soc Neurosci Abstr 13:430.

Berman N, Cynader M (1975) Receptive fields in cat superior colliculus after visual cortex lesions. J Physiol (Lond) 245:261-270.

Berson DM, McIlwain JT (1982) Retinal Y-cell activation of deeplayer cells in superior colliculus of cat. J Neurophysiol 47:700-714.

Capuano U, McIlwain JT (1981) Reciprocity of receptive field images and point images in the superior colliculus of the cat. J Comp Neurol 196:13-23.

Casagrande VA, Diamond IT (1974) Ablation study of the superior colliculus in the tree shrew (Tupaia glis). J Comp Neurol 156:207238.

Casagrande VA, Harting JK, Hall WC, Diamond IT, Martin GF (1972) Superior colliculus of the tree shrew. A structural and functional subdivision into superficial and deep layers. Science 177:444-447.

Chalupa LM, Rhoades RW (1977) Responses of visual, somatosensory and auditory neurons in the golden hamster's superior colliculus. J Physiol (Lond) 270:595-626.

Clemo HR, Stein BE (1984) Topographic organization of somatosensory corticotectal influences in cat. J Neurophysiol 51:843-858.

Clemo HR, Stein BE (1986) Effects of cooling somatosensory cortex on response properties of tactile cells in the superior colliculus. $J$ Neurophysiol 55:1352-1368.

Drager IJC, Hubel DH (1975) Responses to visual stimulation and relationship between visual, auditory and somatosensory inputs in mouse superior colliculus. J Neurophysiol 38:690-713.

Dunning DD, McHaffie JG, Stein BE (1987) A comparison of MAO and $\mathrm{AChE}$ staining patterns in the midbrain with special reference to the superior colliculus. Soc Neurosci Abstr 13:431.

Feldon SP, Feldon P, Kruger L (1970) Topography of the retinal projection upon the superior colliculus of the cat. Vision Res 10:135143.

Finlay BL, Schneps SE, Wilson KG, Schneider GE (1978) Topography of visual and somatosensory projections to the superior colliculus in the golden hamster. Brain Res 142:223-235.

Gilbert CD, Kelly JP (1975) The projections of cells in different layers of the cat's visual cortex. J Comp Neurol 163:81-106.

Gordon BG (1973) Receptive fields in the deep layers of the cat superior colliculus. J Neurophysiol 36:157-178.

Graham J, Pearson HD, Berman N, Murphy EH (1981) Laminar organization of superior colliculus in rabbit: a study of receptive field properties of single units. J Neurophysiol 45:915-932. 
Graybiel AM (1975) Anatomical organization of retinotectal afferents in the cat: an autoradiographic study. Brain Res 96:1-23.

Hardy SC, Stein BE (1988) Small lateral suprasylvian cortex lesions produce visual neglect and decreased visual activity in the superior colliculus. J Comp Neurol 273:527-542.

Harting JK, Guillery RW (1976) Organization of retinocollicular pathways in the cat. J Comp Neurol 166:133-144.

Hartline PH (1984) The optic tectum of reptiles: neurophysiological studies. In: The comparative neurology of the optic tectum (Vanegas H, ed), pp 601-618. New York: Plenum.

Hubel DH, Wiesel TN (1969) Visual area of the lateral suprasylvian gyrus (Claire-Bishop area) of the cat. J Physiol (Lond) 202:251-260.

Huerta MF, Harting JK (1984) The mammalian superior colliculus: studies of morphology and connections. In: The comparative neurology of the optic tectum (Vanegas H, ed), pp 687-774. New York: Plenum.

Jay MF, Sparks DL (1987) Sensorimotor integration in the primate superior colliculus: II. Coordinates of auditory signals. J Neurophysiol 57:35-55.

Kawamura S, Sprague JM, Niimi K (1974) Corticofugal projections from the visual cortices to the thalamus, pretectum, and superior colliculus in the cat. J Comp Neurol 158:339-362.

King AJ, Palmer AR (1983) Cells responsive to free-field auditory stimuli in guinea-pig superior colliculus: distribution and response properties. J Physiol (Lond) 342:361-381.

King AJ, Palmer AR (1985) Integration of visual and auditory information in bimodal neurones in the guinea-pig superior colliculus. Exp Brain Res 60:492-500.

King AI, Hutchings ME, Moore DR, Blakemore C (1988) Developmental plasticity in the visual and auditory representations in the mammalian superior colliculus. Nature 332:73-76.

Knudsen EI (1982) Auditory and visual maps of space in the optic tectum of the owl. J Neurosci 2:1177-1194.

Knudsen EI (1987) Visual deprivation degrades the auditory map of space in the optic tectum of developing barn owls. Soc Neurosci Abstr 13:80.

Knudsen EI, Knudsen PF (1985) Vision guides the adjustment of auditory localization in young barn owls. Science 230:545-548.

Lee C, Rohrer WH, Sparks DL (1988) Population coding of saccadic eye movements by neurons in the superior colliculus. Nature 332: 357-360.

Marzi CA, Antonini A, Di Stefano M, Legg CR (1982) The contribution of the corpus callosum to receptive fields in the lateral suprasylvian visual areas of the cat. Behav Brain Res 4:155-176.

McHaffie JG, Stein BE (1983) A chronic headholder minimizing facial obstructions. Brain Res Bull 10:859-860.

Mcllwain JT (1975) Visual receptive fields and their images in superior colliculus of the cat. J Neurophysiol 38:219-230.

Mcllwain JT (1976) Large receptive fields and spatial transformations in the visual system. In: International review of physiology, neurophysiology II (Porter R, ed), pp 223-248. Baltimore: University Park.

McIlwain JT, Buser P (1968) Receptive fields of single cells in the cat's superior colliculus. Exp Brain Res 5:314-325.

Mcllwain JT, Fields HL (1971) Interaction of cortical and retinal projections on single neurons of the cat's superior colliculus. J Neurophysiol 34:763-772.

Meredith MA, Clemo HR (1989) Auditory cortical projection from the anterior ectosylvian sulcus (field AES) to the superior colliculus in cat: an anatomical and electrophysiological study. J Comp Neurol 289:687-707.

Meredith MA, Stein BE (1986a) Spatial factors determine the activity of multisensory neurons in cat superior colliculus. Brain Res 365: 350-354.

Meredith MA, Stein BE (1986b) Visual, auditory and somatosensory convergence on cells in superior colliculus results in multisensory integration. $\mathrm{J}$ Neurophysiol 56:640-662.

Meredith MA, Stein BE (1988) Multiple visual maps in deep superior colliculus of cats. Soc Neurosci Abstr 14:831.

Meredith MA, Nemitz JW, Stein BE (1987) Determinants of multisensory integration in superior colliculus neurons: I. Temporal factors. J Neurosci 7:3213-3229.

Middlebrooks JC, Knudsen EI (1984) A neural code for auditory space in the cat's superior colliculus. J Neurosci 4:2621-2634.

Mize RR, Murphy EH (1976) Alterations in receptive field properties of superior colliculus cells produced by visual cortex ablation in infant and adult cats. J Comp Neurol 168:393-424.

Mooney RD, Nikoletseas MM, Hess PR, Allen Z, Lewin AC, Rhoades RW (1988a) The projection from the superficial to the deep layers of the superior colliculus: an intracellular horseradish peroxidase injection study in the hamster. J Neurosci 8:1384-1399.

Mooney RD, Fish SE, Nikoletseas MM, Rohrer WH, Rhoades RW (1988b) Intralaminar projections in the hamster's superior colliculus. Soc Neurosci Abstr 14:830.

Morrison DF (1976) Multivariate statistical methods, 2d ed. New York: McGraw-Hill.

Moschovakis AK, Karabelas AB (1985) Observations on the somatodendritic morphology and axonal trajectory of intracellularly HRPlabeled efferent neurons located in the deeper layers of the superior colliculus of the cat. J Comp Neurol 239:276-308.

Moschovakis AK, Karabelas AB, Highstein SM (1988) Structurefunction relationships in the primate superior colliculus. I. Morphological classification of efferent neurons. J Neurophysiol 60:232-262.

Mucke LM, Norita M, Benedek G, Creutzfeldt O (1982) Physiologic and anatomic investigation of a visual cortical area situated in the ventral bank of the anterior ectosylvian sulcus of the cat. Exp Brain Res 179:1-11.

Ogasawara K, McHaffie JG, Stein BE (1984) Two visual corticotectal systems in cat. J Neurophysiol 52:1226-1245.

Olson CR, Graybiel AM (1987) Ectosylvian visual area of the cat: location, retinotopic organization, and connections. J Comp Neurol 261:277-294

Rhoades RW, Mooney RD, Rohrer WH, Nikoletseas MM, Fish SE (1989) Organization of the projection from the superficial to the deep layers of the hamster's superior colliculus as demonstrated by the anterograde transport of Phaseolus vulgaris leucoagglutinin. J Comp Neurol 283:54-70.

Rosenquist AC, Palmer LA (1971) Visual receptive field properties of cells of the superior colliculus after cortical lesions in the cat. Exp Neurol 33:629-652.

Rusoff AC, Dubin MW (1977) Development of receptive-field properties of retinal ganglion cells in kittens. J Neurophysiol 40:11881198.

Segal RL, Beckstead RM (1984) The lateral suprasylvian corticotectal projection in cats. J Comp Neurol 225:259-275.

Sherman M (1974) Visual fields of cats with cortical and tectal lesions. Science 185:355-357.

Spear PD, Baumann TP (1975) Receptive field characteristics of single neurons in lateral suprasylvian visual area of the cat. J Neurophysiol 38:1403-1420.

Sprague JM (1966) Visual, acoustic and somesthetic deficits in the cat after cortical and midbrain lesions. In: The thalamus (Purpura DD, Yahr M, eds), pp 391-417. New York: Columbia UP.

Sprague JM, Meikle TH Jr (1965) The role of the superior colliculus in visually guided behavior. Exp Neurol 11:115-146.

Stein BE (1984) Development of the superior colliculus. Annu Rev Neurosci 7:95-125.

Stein BE, Arigbede MO (1972) Unimodal and multimodal response properties of neurons in the cat's superior colliculus. Exp Neurol 36: 179-196.

Stein BE, Dixon JP (1978) Properties of superior colliculus neurons in golden hamster. J Comp Neurol 183:269-284.

Stein BE, Magalhaes-Castro B (1975) Effects of neonatal cortical lesions upon the cat superior colliculus. Brain Res 83:480-485.

Stein BE, Magalhaes-Castro B, Kruger I (1976) Relationship between visual and tactile representation in cat superior colliculus. J Neurophysiol 39:401-419.

Stcin BE, Spcncer RF, Edwards SB (1983) Corticotectal and corticothalamic efferent projections of SIV somatosensory cortex in cat. J Neurophysiol 50:896-908.

Tiao Y-C, Blakemore C (1976) Functional organization in the superior colliculus of the golden hamster. J Comp Neurol 168:483-506.

Van Gisbergen JAM, Van Opstal AJ, Tax AAM (1987) Collicular ensemble coding of saccades based on vector summation. Neuroscience 21:541-555.

Wickelgren BG, Sterling P (1969) Influence of visual cortex on receptive fields in the superior colliculus of the cat. J Neurophysiol 32:1623. 